\title{
The GalMer database: galaxy mergers in the virtual observatory ${ }^{\star}$
}

\author{
I. V. Chilingarian ${ }^{1,2,3}$, P. Di Matteo ${ }^{4}$, F. Combes ${ }^{1}$, A.-L. Melchior ${ }^{1,5}$, and B. Semelin ${ }^{1,5}$ \\ 1 Observatoire de Paris, LERMA, CNRS UMR 8112, 61 Av. de l'Observatoire, 75014 Paris, France \\ e-mail: igor.chilingarian@obspm.fr \\ 2 Sternberg Astronomical Institute, Moscow State University, 13 Universitetski prospect, 119992 Moscow, Russia \\ 3 Centre de données astronomiques de Strasbourg, Observatoire astronomique de Strasbourg, UMR 7550, Université de Strasbourg / \\ CNRS, 11 rue de l'Université, 67000 Strasbourg, France \\ 4 Observatoire de Paris-Meudon, GEPI, CNRS UMR 8111, 5 pl. Jules Janssen, 92195 Meudon, France \\ 5 Université Pierre et Marie Curie-Paris 6, 4 Place Jussieu, 75252 Paris Cedex 05, France
}

Received 20 June 2009 / Accepted 27 February 2010

\begin{abstract}
We present the GalMer database, a library of galaxy merger simulations, that has been produced and made available to users by means of tools compatible with the Virtual Observatory (VO) standards adapted specially for this theoretical database. To investigate the physics of galaxy formation through hierarchical merging, it is necessary to simulate galaxy interactions varying a large number of parameters, e.g. morphological types, mass ratios, orbital configurations. On the one hand, these simulations have to be performed in a cosmological context, capable of providing a large number of galaxy pairs, with boundary conditions given by the large-scale simulation. On the other hand, the resolution has to be high enough on galaxy scales, to provide realistic physics. The GalMer database is a library of thousands of simulations of galaxy mergers at moderate spatial resolution and represents a compromise between considering a diverse range of initial conditions and optimising the details of underlying physics. We provide all coordinates and data of simulated particles in FITS binary tables. The main advantages of the database are VO access interfaces and valueadded services that allow users to compare the results of the simulations directly to observations: stellar population modelling, dust extinction, spectra, images, visualisation using dedicated VO tools. The GalMer value-added services can be used as a virtual telescope producing broadband images, 1D spectra, 3D spectral datacubes, thus enhancing the utility of our database to observers. We present several examples of the GalMer database scientific usage obtained by analyzing simulations and modelling their stellar population properties, including: (1) studies of the star formation efficiency in interactions; (2) creation of old counter-rotating components; (3) reshaping metallicity profiles in elliptical galaxies; (4) orbital to internal angular momentum transfer; (5) reproducing observed colour bimodality of galaxies.
\end{abstract}

Key words. galaxies: interactions - galaxies: kinematics and dynamics - galaxies: stellar content - methods: numerical astronomical data bases: miscellaneous

\section{Introduction}

In the framework of the present cosmological paradigm, mergers and interactions are among the most important mechanisms governing galaxy formation and evolution. Spitzer \& Baade (1951) proposed that collisions of late-type disc galaxies should produce early-type ones. A natural consequence of this phenomenon is the morphology-density relation discovered three decades later (Dressler 1980): dense regions of the Universe, where galaxy collisions are supposed to be more frequent, contain larger fractions of early-type galaxies than sparsely populated areas.

Toomre \& Toomre (1972) were the first to suggest that interactions "tend to bring deep into a galaxy a fairly sudden supply of fresh fuel in the form of interstellar material..." The gas in the bar formed during the interaction loses its angular momentum due to the torques (Combes et al. 1990; Barnes \& Hernquist 1996), falls onto the galaxy centre possibly inducing strong bursts of star formation (Mihos \& Hernquist 1994a, 1996), and creates a young compact central stellar component (Mihos \& Hernquist 1994b) often observed in present-day early-type

* The GalMer database is accessible online at http: //galmer.obspm. fr/ galaxies (Sil'chenko 2006; Kuntschner et al. 2006). Intense star formation episodes accompanied by supernova explosions, enrich the interstellar medium (ISM) with heavy elements, which are consumed into the stars hence increasing the observed metal abundances of the stellar population.

Large-scale cosmological $N$-body simulations (e.g., Springel et al. 2006; Ocvirk et al. 2008) often lack the spatial resolution to trace in detail star formation and morphological transformation on a galaxy scale. Therefore, they are usually complemented by semi-analytical prescriptions qualitatively accounting for phenomena strongly affecting galaxy evolution, such as star formation (e.g., Blaizot et al. 2004; Somerville et al. 2008). However, the parameters of the semi-analytical models have to be chosen based on more detailed simulations of galaxy interactions. High resolution galaxy simulations (e.g. Bournaud et al. 2008) cannot be performed in large statistical numbers. A compromise has then to be made between statistics and resolution. This becomes one of the main motivations for studying large numbers of galaxy interactions by means of dedicated intermediateresolution numerical simulations.

Merger-induced star formation, as well as morphological transformation, strongly depends on the mass ratio of the interacting galaxies. In general, the intensity of starbursts decreases as the merger mass ratio increases (e.g., Cox et al. 2008). 
Equal-mass mergers of disc galaxies (mass ratios below 4:1) usually result in early-type elliptical-like remnants (Toomre 1977; Naab \& Burkert 2003), while minor mergers (ratios above 10:1) do not destroy the progenitor's disc preserving its exponential mass distribution although making it thicker and dynamically hotter (Quinn et al. 1993; Walker et al. 1996; Velazquez \& White 1999; Bournaud et al. 2005). A sequence of repeated minor mergers can form elliptical galaxies, with global morphological and kinematical properties similar to those observed in real ellipticals (Bournaud et al. 2007).

Orbital parameters of the interaction and orientation of galaxies also strongly affect the process of merging, e.g., star formation efficiency on retrograde orbits is generally higher than for direct encounters (Di Matteo et al. 2007).

One needs to explore a large multi-dimensional parameter space (different initial morphologies related to gas content, orbital configurations, mass ratios, etc.) by running thousands of simulations to fully understand the astrophysical consequences of galaxy interactions for the modern picture of galaxy evolution.

The GalMer project, developed in the framework of the French national HORIZON ${ }^{1}$ collaboration, has the ambitious goal of providing access for the astronomical community to the results of massive intermediate-resolution numerical simulations of galaxy interactions in pairs, covering as much as possible the parameter space of initial conditions and, thus allowing us to study statistically the star formation enhancements, and structural and dynamical properties of merger remnants. An important aspect is the integration with the data services available in the framework of the International Virtual Observatory to take full advantage of already developed technologies and data visualisation and processing tools.

This paper is a technical presentation of: (1) the GalMer TreeSPH simulations providing all essential details about the initial conditions for galaxies of different masses and prescriptions used to model the processes of star formation including supernova feedback and metallicity evolution; (2) the GalMer database, the first VO resource containing results of TreeSPH simulations; (3) the GalMer value-added services aimed at facilitating the comparison between simulations and observations such as modelling the spectrophotometric galaxy properties using an evolutionary synthesis code.

The paper is organised as follows: in Sect. 2, we describe initial conditions of the numerical simulations and orbital parameters of galaxy interactions; Sect. 3 contains information about the numerical method, and prescriptions for both star formation and metallicity evolution; Sect. 4 describes the GalMer database structure, its access interface, and mechanisms of data visualisation; Sect. 5 presents value-added services of the GalMer database; in Sect. 6, we define some astrophysical applications that can be tackled with our simulations; Sect. 7 contains a brief summary.

\section{Initial conditions}

\subsection{Galaxy models: moving along the Hubble sequence}

We model interactions among galaxies of different morphologies, from ellipticals to late-type spirals. The adopted galaxy models consist of a spherical non-rotating dark-matter halo, which may or may not contain a stellar and a gaseous disc and, optionally, a central non-rotating bulge. For each galaxy type, the

1 http://www.projet-horizon.fr/ halo and the optional bulge are modelled as Plummer spheres, with characteristic masses $M_{\mathrm{B}}$ and $M_{\mathrm{H}}$ and characteristic radii $r_{\mathrm{B}}$ and $r_{\mathrm{H}}$. Our choice of adopting a core density distribution for the dark halo seems to be more in accordance with the rotation curves of local spirals and dwarf galaxies, than the cuspy profiles predicted by Cold Dark Matter simulations (see Di Matteo et al. 2008a, Sect. 2.4.3 for a discussion). The stellar and gaseous discs follow the Miyamoto \& Nagai (1975) density profile, with masses $M_{*}$ and $M_{\mathrm{g}}$ and vertical and radial scale lengths given, respectively, by $h_{*}$ and $a_{*}$, and $h_{\mathrm{g}}$ and $a_{\mathrm{g}}$. We refer the reader to Appendix A for the analytical expression of these profiles. For the initial models of the disc galaxies, we chose an initial Toomre parameter for the stellar disc $Q_{\text {star }}=1.2$, and two different initial ones for the gas component, $Q_{\mathrm{gas}}=0.3$ and $Q_{\mathrm{gas}}=1.2$, to reproduce, at least partially, the variety of parameters found in real galaxies (Martin \& Kennicutt 2001; Boissier et al. 2003; Hitschfeld et al. 2009).

The database contains interacting galaxy pairs of different mass ratios (1:1, 1:2, 1:10), involving a giant galaxy (gE0 for a giant-like elliptical, gS0 for a giant-like lenticular, gSa for a giant-like Sa spiral, gSb for a giant-like Sbc spiral, and gSd for a giant-like Sd spiral), interacting with:

- either another giant galaxy;

- or an intermediate-mass galaxy (hereafter iE0, iS0, iSa, iSb and iSd), of a total mass half that of the giant's mass;

- or a dwarf galaxy (hereafter $\mathrm{dE} 0, \mathrm{dS} 0, \mathrm{dSa}, \mathrm{dSb}$ and $\mathrm{dSd}$ ) whose total mass is ten times lower than that of the giant galaxy.

The complete Hubble sequence of the galaxy models is given in Fig. 1. Moving along the Hubble sequence, from gE0 to gSd galaxies, the mass of the central spheroid varies from $M_{\mathrm{B}}=$ $1.6 \times 10^{11} M_{\odot}$ for a $\mathrm{gE0}$ to $M_{\mathrm{B}}=0$ for a $\mathrm{gSd}$, while the gas mass $M_{g}$, absent in the case of a gE0 and gS0, increases from $9.2 \times 10^{9} M_{\odot}$ in a gSa to $1.7 \times 10^{10} M_{\odot}$ for a gSd (see Table 1 for a complete list of all the parameters of giant-like galaxies, and Tables 2 and 3 for the corresponding set of parameters of intermediate and dwarf systems). Our modelled Hubble sequence capture the main properties of local galaxies. In the future we plan to extend this library to higher redshift, considering larger gas fractions for the disc galaxies.

Each galaxy consists of a total number of particles $N_{\text {TOT }}$ distributed among gas, stars and dark matter. Gas particles are actually "hybrid particles", characterized by two mass values: one corresponds to the gravitational mass and remains unchanged during the whole simulation, and the other is the gas content of the particle, decreasing or increasing according to the local star formation rate and mass loss (see Sect. 3.1, Eq. (1)). When the gas fraction is below a certain threshold, the particle is transformed into a star particle, and the remaining gas mass is distributed over neighbouring gas particles.

For giant-giant interactions, each galaxy consists of a total number of particles of $N_{\text {TOT }}=120000$, distributed among gas, stars and dark matter, depending on the morphology (Table 4). For giant-intermediate and giant-dwarf interactions, we increased the total number of particles by a factor 4 in the giant galaxy. This allowed us to improve the spatial resolution of the simulations and, in particular, to maintain a high enough numerical resolution for the smaller galaxy $\left(N_{\mathrm{TOT}}=240000\right.$ and $N_{\text {TOT }}=48000$ for the intermediate and dwarf galaxy, respectively; Tables 5 and 6).

The initial rotation curves of the modelled disc galaxies are shown in Fig. 2. 


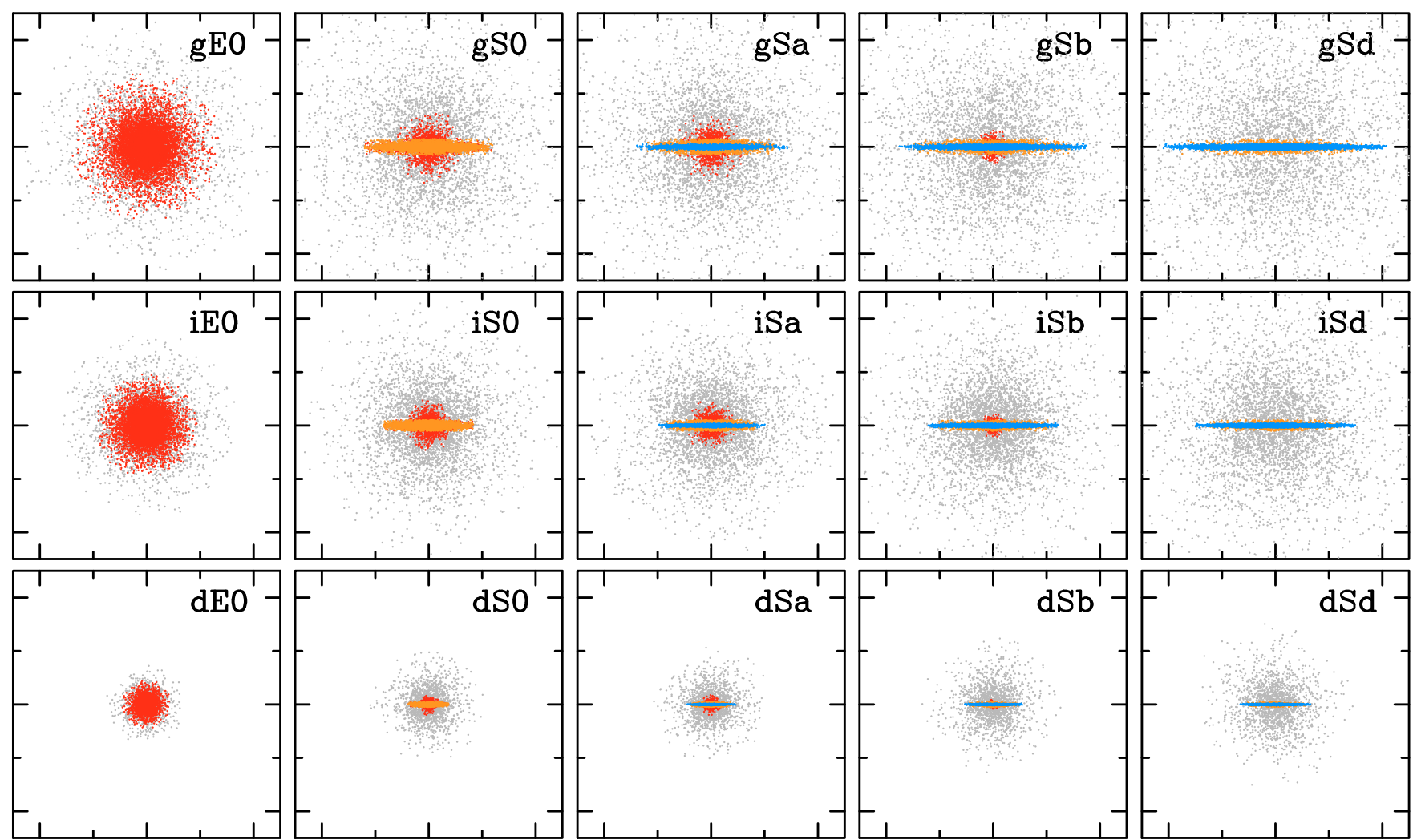

Fig. 1. Hubble sequence of the GalMer galaxy models. From left to right, and from the top to the bottom, projection on the $x$ - $z$ plane of giant, intermediate and dwarf galaxies. Different colors correspond to the different galaxy components: dark matter (grey), bulge (red), stellar disc (orange), and gaseous disc (blue). Each frame is $50 \mathrm{kpc} \times 50 \mathrm{kpc}$ in size.

Table 1. Galaxy parameters for giant galaxies.

\begin{tabular}{|c|c|c|c|c|c|}
\hline & gE0 & gS0 & $\mathrm{gSa}$ & $\mathrm{gSb}$ & gSd \\
\hline$M_{\mathrm{B}}\left[2.3 \times 10^{9} M_{\odot}\right]$ & 70 & 10 & 10 & 5 & 0 \\
\hline$M_{\mathrm{H}}\left[2.3 \times 10^{9} M_{\odot}\right]$ & 30 & 50 & 50 & 75 & 75 \\
\hline$M_{*}\left[2.3 \times 10^{9} M_{\odot}\right]$ & 0 & 40 & 40 & 20 & 25 \\
\hline$M_{\mathrm{g}} / M_{*}$ & - & - & 0.1 & 0.2 & 0.3 \\
\hline$r_{\mathrm{B}}[\mathrm{kpc}]$ & 4 & 2 & 2 & 1 & - \\
\hline$r_{\mathrm{H}}[\mathrm{kpc}]$ & 7 & 10 & 10 & 12 & 15 \\
\hline$a_{*}[\mathrm{kpc}]$ & - & 4 & 4 & 5 & 6 \\
\hline$h_{*}[\mathrm{kpc}]$ & - & 0.5 & 0.5 & 0.5 & 0.5 \\
\hline$a_{\mathrm{g}}[\mathrm{kpc}]$ & - & - & 5 & 6 & 7 \\
\hline$h_{\mathrm{g}}[\mathrm{kpc}]$ & - & - & 0.2 & 0.2 & 0.2 \\
\hline
\end{tabular}

To initialize particle velocities, we adopted the method described in Hernquist (1993).

To differentiate the role of interactions from secular evolution, for each galaxy model we ialso provide in the database its evolution in isolation, for 3 Gyr. A brief description of how the modeled galaxies evolve in isolation is given in Appendix B.

\subsection{Orbital parameters}

For each pair of interacting galaxies, we performed several simulations, varying the galaxies' orbital initial conditions (initial orbital energy $E$ and angular momentum $L$ ) and taking into account both direct and retrograde orbits (Tables 7-9). For each interacting pair, we kept the disc (when present) of one of the two galaxies in the orbital plane $\left(i_{1}=0^{\circ}\right)$, and varied the inclination $i_{2}$ of the companion disc, considering $i_{2}=0^{\circ}, i_{2}=45^{\circ}, i_{2}=75^{\circ}$,
Table 2. Galaxy parameters for intermediate galaxies.

\begin{tabular}{|c|c|c|c|c|c|}
\hline & $\mathrm{iE} 0$ & iS0 & $\mathrm{iSa}$ & $\mathrm{iSb}$ & iSd \\
\hline$M_{\mathrm{B}}\left[2.3 \times 10^{9} M_{\odot}\right]$ & 35 & 5 & 5 & 2.5 & 0 \\
\hline$M_{\mathrm{H}}\left[2.3 \times 10^{9} M_{\odot}\right]$ & 15 & 25 & 25 & 37.5 & 37.5 \\
\hline$M_{*}\left[2.3 \times 10^{9} M_{\odot}\right]$ & 0 & 20 & 20 & 10 & 12.5 \\
\hline$M_{\mathrm{g}} / M_{*}$ & - & - & 0.1 & 0.2 & 0.3 \\
\hline$r_{\mathrm{B}}[\mathrm{kpc}]$ & 2.8 & 1.4 & 1.4 & 0.7 & - \\
\hline$r_{\mathrm{H}}[\mathrm{kpc}]$ & 5. & 7. & 7. & 8.5 & 10.6 \\
\hline$a_{*}[\mathrm{kpc}]$ & - & 2.8 & 2.8 & 3.5 & 4.2 \\
\hline$h_{*}[\mathrm{kpc}]$ & - & 0.35 & 0.35 & 0.35 & 0.35 \\
\hline$a_{\mathrm{g}}[\mathrm{kpc}]$ & - & - & 3.5 & 4.2 & 5. \\
\hline$h_{\mathrm{g}}[\mathrm{kpc}]$ & - & - & 0.14 & 0.14 & 0.14 \\
\hline
\end{tabular}

and $i_{2}=90^{\circ}$. The clustering of the angles toward $i_{2}=90^{\circ}$, for an uneven sampling, is logical from a purely geometrical point of view, considering that the probability of the spin $i_{2}$ of the second galaxy to be oriented between 0 and $i_{2}$ is proportional to $1-\cos \left(i_{2}\right)$. This means, for example, that an orientation $i_{2}$ between $45^{\circ}$ and $90^{\circ}$ has a probability 2.3 times higher than an orientation $i_{2}$ between $0^{\circ}$ and $45^{\circ}$. However, the spin alignment may not be totally uncorrelated, as shown by Jimenez et al. (2010), but a distribution function, to our knowledge, is still lacking. For giant-dwarf interactions, we also consider a more generic case, with $i_{1}=33^{\circ}$, and $i_{2}=130^{\circ}$ (see Fig. 3 for a sketch of the initial orbital geometry and Table 10 for the orientation of the galaxy spins). 
Table 3. Galaxy parameters for dwarf galaxies.

\begin{tabular}{|c|c|c|c|c|c|}
\hline & $\mathrm{dE} 0$ & dS0 & $\mathrm{dSa}$ & $\mathrm{dSb}$ & $\mathrm{dSd}$ \\
\hline$M_{\mathrm{B}}\left[2.3 \times 10^{9} M_{\odot}\right]$ & 7 & 1 & 1 & 0.5 & 0 \\
\hline$M_{\mathrm{H}}\left[2.3 \times 10^{9} M_{\odot}\right]$ & 3 & 5 & 5 & 7.5 & 7.5 \\
\hline$M_{*}\left[2.3 \times 10^{9} M_{\odot}\right]$ & 0 & 4 & 4 & 2 & 2.5 \\
\hline$M_{\mathrm{g}} / M_{*}$ & - & - & 0.1 & 0.2 & 0.3 \\
\hline$r_{\mathrm{B}}[\mathrm{kpc}]$ & 1.3 & 0.6 & 0.6 & 0.3 & - \\
\hline$r_{\mathrm{H}}[\mathrm{kpc}]$ & 2.2 & 3.2 & 3.2 & 3.8 & 4.7 \\
\hline$a_{*}[\mathrm{kpc}]$ & - & 1.3 & 1.3 & 1.6 & 1.9 \\
\hline$h_{*}[\mathrm{kpc}]$ & - & 0.16 & 0.16 & 0.16 & 0.16 \\
\hline$a_{\mathrm{g}}[\mathrm{kpc}]$ & - & - & 1.6 & 1.9 & 2.2 \\
\hline$h_{\mathrm{g}}[\mathrm{kpc}]$ & - & - & 0.06 & 0.06 & 0.06 \\
\hline
\end{tabular}

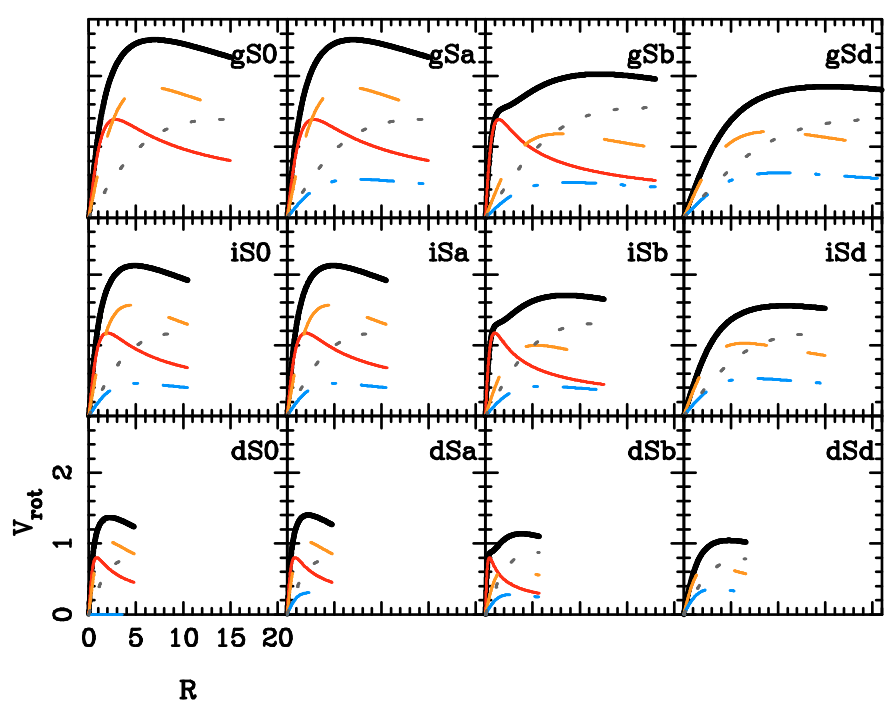

Fig. 2. Rotation curves for the different galaxy models. The initial rotation curve is shown (solid thick black curve), together with the contribution of the bulge component (thin solid red curve), the stellar disc (orange dashed curve), the gaseous disc (dot-dashed blue curve), and the dark matter halo (dotted grey curve). Lengths are in kpc and velocities in units of $100 \mathrm{~km} \mathrm{~s}^{-1}$.

Table 4. Particle numbers for giant-giant interactions (mass ratio 1:1).

\begin{tabular}{cccccc}
\hline \hline & $\mathrm{gE0}$ & $\mathrm{gS0}$ & $\mathrm{gSa}$ & $\mathrm{gSb}$ & $\mathrm{gSd}$ \\
\hline$N_{\text {gas }}$ & - & - & 20000 & 40000 & 60000 \\
$N_{\text {star }}$ & 80000 & 80000 & 60000 & 40000 & 20000 \\
$N_{\text {DM }}$ & 40000 & 40000 & 40000 & 40000 & 40000 \\
\hline
\end{tabular}

\section{Numerical method}

To model galaxy evolution, we employed a Tree-SPH code, in which gravitational forces are calculated using a hierarchical tree method (Barnes \& Hut 1986) and gas evolution is followed by means of smoothed particle hydrodynamics (SPH, Lucy 1977; Gingold \& Monaghan 1982). Gravitational forces are calculated using a tolerance parameter $\theta=0.7$ and include terms up to the quadrupole order in the multipole expansion. A Plummer potential is used to soften gravitational forces, similar softening lengths being adopted for all particle types. We assume a softening length $\epsilon=280 \mathrm{pc}$ for giant-giant interactions, and $\epsilon=200 \mathrm{pc}$ for the giant-intermediate and giant-dwarf runs.

The code (i.e. its evaluation of the gravitational forces, and implementation of the SPH technique and star formation modelling) was described in Semelin \& Combes (2002), where
Table 5. Particle numbers for giant-intermediate interactions (mass ratio $1: 2)$.

\begin{tabular}{cccccc}
\hline \hline & gE0 & gS0 & gSa & gSb & gSd \\
\hline$N_{\text {gas }}$ & - & - & 80000 & 160000 & 240000 \\
$N_{\text {star }}$ & 320000 & 320000 & 240000 & 160000 & 80000 \\
$N_{\text {DM }}$ & 160000 & 160000 & 160000 & 160000 & 160000 \\
\hline \hline & iE0 & iS0 & iSa & iSb & iSd \\
\hline$N_{\text {gas }}$ & - & - & 40000 & 80000 & 120000 \\
$N_{\text {star }}$ & 160000 & 160000 & 120000 & 80000 & 40000 \\
$N_{\text {DM }}$ & 80000 & 80000 & 80000 & 80000 & 80000 \\
\hline
\end{tabular}

Table 6. Particle numbers for giant-dwarf interactions (mass ratio 1:10).

\begin{tabular}{cccccc}
\hline \hline & $\mathrm{gE0}$ & $\mathrm{gS} 0$ & $\mathrm{gSa}$ & $\mathrm{gSb}$ & $\mathrm{gSd}$ \\
\hline$N_{\text {gas }}$ & - & - & 80000 & 160000 & 240000 \\
$N_{\text {star }}$ & 320000 & 320000 & 240000 & 160000 & 80000 \\
$N_{\text {DM }}$ & 160000 & 160000 & 160000 & 160000 & 160000 \\
\hline \hline & $\mathrm{dE0}$ & $\mathrm{dS} 0$ & $\mathrm{dSa}$ & $\mathrm{dSb}$ & $\mathrm{dSd}$ \\
\hline$N_{\text {gas }}$ & - & - & 8000 & 16000 & 24000 \\
$N_{\text {star }}$ & 32000 & 32000 & 24000 & 16000 & 8000 \\
$N_{\text {DM }}$ & 16000 & 16000 & 16000 & 16000 & 16000 \\
\hline
\end{tabular}

Table 7. Orbital parameters for giant-giant interactions.

\begin{tabular}{|c|c|c|c|c|c|}
\hline orb.id & $\begin{array}{l}r_{\mathrm{ini}}{ }^{a} \\
\mathrm{kpc}\end{array}$ & $\begin{array}{c}v_{\mathrm{ini}}^{b} \\
10^{2} \mathrm{~km} \mathrm{~s}^{-1} \\
\end{array}$ & $\begin{array}{c}L^{c} \\
10^{2} \mathrm{~km} \mathrm{~s}^{-1} \mathrm{kpc} \\
\end{array}$ & $\begin{array}{c}E^{d} \\
10^{4} \mathrm{~km}^{2} \mathrm{~s}^{-2} \\
\end{array}$ & $\operatorname{spin}^{e}$ \\
\hline 01dir & 100. & 2. & 56.6 & 0.0 & up \\
\hline 01ret & 100. & 2. & 56.6 & 0.0 & down \\
\hline 02dir & 100. & 3. & 59.3 & 2.5 & up \\
\hline 02ret & 100. & 3. & 59.3 & 2.5 & down \\
\hline 03dir & 100. & 3.7 & 62.0 & 5.0 & up \\
\hline 03ret & 100. & 3.7 & 62.0 & 5.0 & down \\
\hline 04dir & 100. & 5.8 & 71.5 & 15.0 & up \\
\hline 04ret & 100. & 5.8 & 71.5 & 15.0 & down \\
\hline 05dir & 100. & 2. & 80.0 & 0.0 & up \\
\hline 05ret & 100. & 2. & 80.0 & 0.0 & down \\
\hline 06dir & 100. & 3. & 87.6 & 2.5 & up \\
\hline 06ret & 100. & 3. & 87.6 & 2.5 & down \\
\hline 07dir & 100. & 3.7 & 94.6 & 5.0 & up \\
\hline 07ret & 100. & 3.7 & 94.6 & 5.0 & down \\
\hline 08dir & 100. & 5.8 & 118.6 & 15.0 & up \\
\hline 08ret & 100. & 5.8 & 118.6 & 15.0 & down \\
\hline 09dir & 100. & 2.0 & 97.9 & 0.0 & up \\
\hline 09ret & 100. & 2.0 & 97.9 & 0.0 & down \\
\hline 10dir & 100. & 3.0 & 111.7 & 2.5 & up \\
\hline 10ret & 100. & 3.0 & 111.7 & 2.5 & down \\
\hline 11dir & 100. & 3.7 & 123.9 & 5.0 & up \\
\hline 11ret & 100. & 3.7 & 123.9 & 5.0 & down \\
\hline 12dir & 100. & 5.8 & 163.9 & 15.0 & up \\
\hline 12ret & 100. & 5.8 & 163.9 & 15.0 & down \\
\hline
\end{tabular}

Notes. ${ }^{(a)}$ Initial distance between the two galaxies; ${ }^{(b)}$ absolute value of the initial relative velocity; ${ }^{(c)} L=\left|\boldsymbol{r}_{\text {ini }} \times v_{\text {ini }}\right| ;{ }^{(d)} E=v_{\text {ini }}{ }^{2} / 2-$ $G\left(m_{1}+m_{2}\right) / r_{\text {ini }}$, with $m_{1}=m_{2}=2.3 \times 10^{11} M_{\odot} ;{ }^{(e)}$ orbital spin, if the $z$-component is parallel (up) or anti-parallel (down) to the $z$-axis.

a standard validation test for this type of code (the collapse of an initially static, isothermal sphere of self-gravitating gas, see Evrard (1988) and also Hernquist \& Katz (1989); Thacker et al. (2000) and Springel et al. 2001) was also presented. In our present study, we adopted an isothermal gas phase for the GalMer runs. Other tests are presented in Di Matteo et al. (2008a). 
Table 8. Orbital parameters for giant-intermediate interactions.

\begin{tabular}{cccccc}
\hline \hline orb.id & $\begin{array}{c}r_{\text {ini }}{ }^{a} \\
\mathrm{kpc}\end{array}$ & $\begin{array}{c}v_{\text {ini }}{ }^{b} \\
10^{2} \mathrm{~km} \mathrm{~s}^{-1}\end{array}$ & $\begin{array}{c}L^{c} \\
10^{2} \mathrm{~km} \mathrm{~s}^{-1} \mathrm{kpc}^{2}\end{array}$ & $\begin{array}{c}E^{d} \\
10^{4} \mathrm{~km}^{2} \mathrm{~s}^{-2}\end{array}$ & $\begin{array}{c}\text { spin } \\
\end{array}$ \\
\hline 01dir & 100. & 1.73 & 48.0 & 0.0 & up \\
01ret & 100. & 1.73 & 48.0 & 0.0 & down \\
02dir & 100. & 1.79 & 49.0 & 0.1 & up \\
02ret & 100. & 1.79 & 49.0 & 0.1 & down \\
03dir & 100. & 1.73 & 60.0 & 0.0 & up \\
03ret & 100. & 1.73 & 60.0 & 0.0 & down \\
04dir & 100. & 1.79 & 61.0 & 0.1 & up \\
04ret & 100. & 1.79 & 61.0 & 0.1 & down \\
05dir & 100. & 1.73 & 69.3 & 0.0 & up \\
05ret & 100. & 1.73 & 69.3 & 0.0 & down \\
06dir & 100. & 1.79 & 69.7 & 0.1 & up \\
06ret & 100. & 1.79 & 69.7 & 0.1 & down \\
\hline
\end{tabular}

Notes. ${ }^{(a)}$ Initial distance between the two galaxies; ${ }^{(b)}$ absolute value of the initial relative velocity; ${ }^{(c)} L=\left|\boldsymbol{r}_{\text {ini }} \times \boldsymbol{v}_{\text {ini }}\right| ;{ }^{(d)} E=v_{\text {ini }}{ }^{2} / 2-G\left(m_{1}+\right.$ $\left.m_{2}\right) / r_{\text {ini }}$, with $m_{1}=2.3 \times 10^{11} M_{\odot}$ and $m_{2}=1.15 \times 10^{10} M_{\odot}$; ${ }^{(e)}$ orbital spin, if the $z$-component is parallel (up) or anti-parallel (down) to the $z$-axis.

Table 9. Orbital parameters for giant-dwarf interactions.

\begin{tabular}{|c|c|c|c|c|c|}
\hline orb.id & $\begin{array}{l}r_{\mathrm{ini}}{ }^{a} \\
\mathrm{kpc}\end{array}$ & $\begin{array}{c}v_{\text {inin }}^{b} \\
10^{2} \mathrm{~km} \mathrm{~s}^{-1}\end{array}$ & $\begin{array}{c}L^{c} \\
10^{2} \mathrm{~km} \mathrm{~s}^{-1} \mathrm{kpc}\end{array}$ & $\begin{array}{c}E^{d} \\
10^{4} \mathrm{~km}^{2} \mathrm{~s}^{-2}\end{array}$ & $\operatorname{spin}^{e}$ \\
\hline 01dir & 100. & 1.48 & 29.66 & 0. & up \\
\hline 01 ret & 100. & 1.48 & 29.66 & 0. & down \\
\hline 02dir & 100. & 1.52 & 29.69 & 0.05 & up \\
\hline 02ret & 100. & 1.52 & 29.69 & 0.05 & down \\
\hline 03dir & 100. & 1.55 & 29.72 & 0.1 & up \\
\hline 03ret & 100. & 1.55 & 29.72 & 0.1 & down \\
\hline 04dir & 100. & 1.48 & 36.33 & 0.0 & up \\
\hline 04ret & 100. & 1.48 & 36.33 & 0.0 & down \\
\hline 05dir & 100. & 1.52 & 36.38 & 0.05 & up \\
\hline 05ret & 100. & 1.52 & 36.38 & 0.05 & down \\
\hline 06dir & 100. & 1.55 & 36.43 & 0.1 & up \\
\hline 06ret & 100. & 1.55 & 36.43 & 0.1 & down \\
\hline
\end{tabular}

Notes. ${ }^{(a)}$ Initial distance between the two galaxies; ${ }^{(b)}$ absolute value of the initial relative velocity; (c) $L=\left|\mathbf{r}_{\text {ini }} \times \mathbf{v}_{\text {ini }}\right| ;{ }^{(d)} E=v_{\text {ini }}{ }^{2} / 2-G\left(m_{1}+\right.$ $\left.m_{2}\right) / r_{\text {ini }}$, with $m_{1}=2.3 \times 10^{11} M_{\odot}$ and $m_{2}=2.3 \times 10^{10} M_{\odot} ;{ }^{(e)}$ orbital spin, if the $z$-component is parallel (up) or anti-parallel (down) to the $z$-axis.

Smoothed Particle Hydrodynamics (SPH) is a Lagrangian technique in which the gas is partitioned into fluid elements represented by particles, which obey equations of motion similar to the collisionless component, but contain additional terms describing pressure gradients, viscous forces and radiative effects in gas. To capture shocks, a conventional form of the artificial viscosity is used, with parameters $\alpha=0.5$ and $\beta=1.0$ (Hernquist \& Katz 1989). To describe different spatial dynamical ranges, SPH particles have individual smoothing lengths $h_{i}$, calculated in such a way that a constant number of neighbours lies within $2 h_{i}$. The giant-giant simulations were performed using a number of neighbours $N_{s} \sim 15$; for the giant-intermediate and giant-dwarf interactions $N_{s} \sim 50$. The gas is modelled as isothermal, with a temperature $T_{\text {gas }}=10^{4} \mathrm{~K}$. Because of the short cooling time of disc gas, fluctuations in the gas temperature are quickly radiated away, so that simulations employing an isothermal equation of state differ little from more realistic ones (Mihos \& Hernquist 1996; Naab et al. 2006). The equations of motion are integrated using a leapfrog algorithm with a fixed time step of $\Delta t=5 \times 10^{5} \mathrm{yr}$.

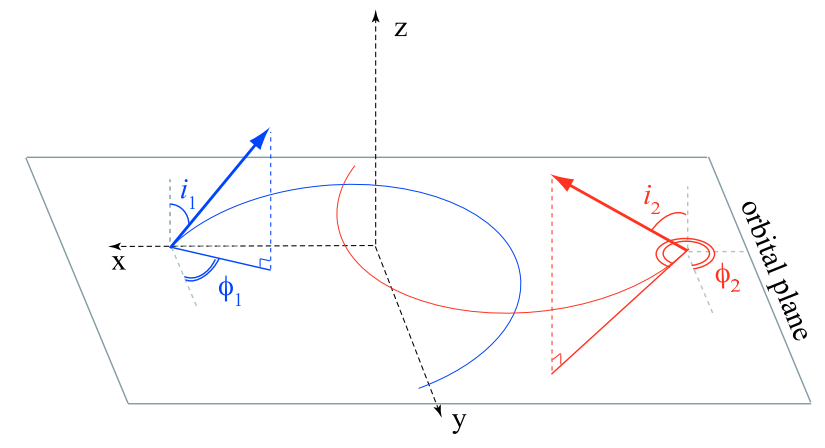

Fig. 3. Adopted orbital geometry for the simulations. We set up the collision in such a way that the orbital angular momentum is parallel to the $z$-axis and the centers of the two galaxies are initially on the $x$-axis. The galaxy spins are represented by the blue and red arrows, respectively. They are specified in terms of the spherical coordinates $\left(i_{1}, \Phi_{1}\right)$ and $\left(i_{2}, \Phi_{2}\right)$. See Table 10 for their initial values.

Table 10. Orientation of the galaxy spins, for giant-giant, giantintermediate, and giant-dwarf simulations.

\begin{tabular}{lcc}
\hline \hline & $\begin{array}{c}\text { giant-giant } \\
\text { giant-intermediate } \\
\text { giant-dwarf }\end{array}$ & giant-dwarf \\
\hline$i_{1}$ & $0^{\circ}$ & $33^{\circ}$ \\
$\Phi_{1}$ & $0^{\circ}$ & $0^{\circ}$ \\
$i_{2}$ & $0^{\circ}, 45^{\circ}, 75^{\circ}, 90^{\circ}$ & $130^{\circ}$ \\
$\Phi_{2}$ & $0^{\circ}$ & $0^{\circ}$ \\
\hline
\end{tabular}

\subsection{Star formation and continuous stellar mass loss}

Numerous prescriptions and techniques exist (Katz 1992; Steinmetz \& Mueller 1994; Springel 2000; Springel \& Hernquist 2003; Cox et al. 2006) for modelling star formation rate and feedback in numerical simulations.

As in Mihos \& Hernquist (1994b), we parametrized the star formation efficiency for a SPH particle as

$\frac{\dot{M}_{\mathrm{gas}}}{M_{\mathrm{gas}}}=C \times \rho_{\mathrm{gas}}{ }^{1 / 2}$,

where the constant $C=0.3 \mathrm{pc}^{3 / 2} M_{\odot}^{-1 / 2} \mathrm{Gyr}^{-1}$ such that the isolated giant disc galaxies form stars at an average rate of between 1 and $2.5 M_{\odot} \mathrm{yr}^{-1}$.

The choice of the parametrization in Eq. (1) is consistent with the observational evidence that on global scales the SFR in disc galaxies is represented well by a Schmidt law of the form $\Sigma_{\mathrm{SFR}}=A \Sigma_{\text {gas }}{ }^{N}, \Sigma_{\text {gas }}$ and $\Sigma_{\mathrm{SFR}}$ being disc-averaged surface densities, with a best fit slope $N$ about 1.4 (see Kennicutt 1998, but also Wong \& Blitz 2002; Boissier et al. 2003; Gao \& Solomon 2004). This relation seems to apply, with a similar slope, also to local scales, as shown in Kennicutt et al. (2005) for Messier 51. As we checked, the prescriptions we adopted reproduce well the Schmidt law in the $\Sigma_{\text {gas }}-\Sigma_{\text {SFR }}$ plane, for both isolated and interacting galaxies (Di Matteo et al. 2007).

Once the SFR recipe is defined, we apply it to SPH particles using the hybrid method described in Mihos \& Hernquist (1994b). In this method, each initial gas particle is hybrid containing a gas fraction and a star fraction. Its gravitational mass $M_{i}$ remains constant, but the gas content of the particle, $M_{i, \mathrm{gas}}$, changes over time according to Eq. (1). Gravitational forces are always evaluated using the gravitational mass $M_{i}$, while hydrodynamical quantities, in turn, use the time-varying $M_{i, \text { gas }}$. If the 
gas fraction present in the hybrid particles drops below $5 \%$ of the initial gas content, the hybrid particle is totally converted into a star-like particle and the small amount of gas material remaining is dispersed between the gas fraction of its neighbours.

The effects of star formation on the surrounding ISM were implemented using the technique described in Mihos \& Hernquist (1994b). For each star-forming hybrid particle, we evaluate the number of stars formed with masses $>8 M_{\odot}$ (a Miller \& Scalo (1979) stellar initial mass function (IMF) is adopted), and we assume that they instantaneously become supernovae, leaving behind remnants of $1.4 M_{\odot}$ and releasing their mass into the surrounding ISM. The released mass also enriches the metallicity of the surrounding gas. This is achieved by assuming a yield $y=M_{\text {ret }} / M_{*}=0.02$, where $M_{\text {ret }}$ is the total mass of all reprocessed metals and $M_{*}$ the total mass in stars. For each gas particle, a return of mass and metals is applied to the $i$-th neighbour gas particle, using a weight $w_{i}$ based on the smoothing kernel.

The energy injection in the ISM from SNe explosions is modelled by assuming that only a fraction $\epsilon_{\mathrm{kin}}$ of $E_{\mathrm{SN}}=10^{51} \mathrm{erg}$ goes into kinetic energy, by applying a radial kick to velocities of neighbouring gas particles; thus, for each $\mathrm{SNe}$ explosion, the $i$ th neighbouring gas particle receives a velocity impulse directed radially away from the "donor"

$\Delta v_{i}=\left(\frac{2 w_{i} \epsilon_{\mathrm{kin}} E_{\mathrm{SN}}}{M_{i}}\right)^{1 / 2}$,

$w_{i}$ being the weighting based on the smoothing kernel and $M_{i}$ the mass of the receiver.

The value of $\epsilon_{\text {kin }}$ is chosen such that the total amount of kinetic energy received by a gas particle, due to the contribution from all its neighbours, is $\leq 1 \mathrm{~km} \mathrm{~s}^{-1}$ to prevent a rapid growth of the vertical thickness of the gaseous disc.

In addition to star formation, we also take into account the competing process of stellar mass-loss: at each time step, an amount

$M_{i, \mathrm{~s}}(t)=\frac{\left(M_{i}-M_{i, \mathrm{gas}}(t)\right) \Delta t c_{0}}{t-t_{\text {birth }}+T_{0}}$

of the stellar mass formed in the hybrid particle is assumed to be lost by evolutionary effects, going to enrich the gas content $M_{i \text {,gas }}$ of the particle. In the formula above, $t_{\text {birth }}$ represents the birth time of the population, $T_{0}=4.97 \mathrm{Myr}$, and $c_{0}=5.47 \times 10^{-2}$ (see Jungwiert et al. 2001, for details).

\subsection{Metallicity evolution}

The metallicity content of the modelled giant galaxies is initially distributed according to a radial profile

$z_{m}(R)=z_{0} \times 10^{-0.07 * R}$,

where $R$ is the particle distance from the galaxy center and $z_{0}=$ $3 \times z_{\odot}$ (Kennicutt et al. 2003; Magrini et al. 2007; Lemasle et al. 2008). For intermediate and dwarf galaxies, we generalised this formula, taken account of a dependency on both the galaxy mass and its half-mass radius (Tremonti et al. 2004; Lee et al. 2006):

$$
\begin{aligned}
z_{m}(R) & =\sqrt{M_{\text {gal }} / M_{\text {giant }}} z_{0} \times 10^{-0.07 * 4.85 * R / r_{50}} \\
& =\sqrt{M_{\text {gal }} / M_{\text {giant }}} z_{0} \times 10^{-0.34 * R / r_{50}},
\end{aligned}
$$

$M_{\text {gal }}$ being the mass of the intermediate (or dwarf galaxy), $r_{50}$ its half-mass radius, $M_{\text {giant }}$ the mass of the giant galaxy, and $r_{50}=4.85 \mathrm{kpc}$ the average half-mass radius of giant galaxies in the sample.

The metallicity of the old stellar component remains unchanged during the simulations, so that only remixing and dynamical effects can reshape the initial gradient of the old stellar population. In turn, the metallicity of both the gas component and the new stellar population (that formed during the simulation) changes with time, because of the release of metals from $\mathrm{SNe}$ explosions, as star formation proceeds. In more detail, hybrid particles are characterised by two metallicity values, $z_{m}$ and $z_{\text {new }}$. The first value corresponds to the metallicity of the gas mass of the particle, while the second one provides the metallicity of the stellar mass contained in the hybrid particle. As for old stars, their metallicity $z_{m}$ is initially distributed in the galaxy disc accordingly to Eq. (4), the central regions being more metal-rich than the outer disc. The metallicity $z_{\text {new }}$ of a new stellar component, is set to be equal to that of the gas in which it forms, while the reprocessed metals enrich the surrounding gas, according to the yield described before.

\section{The GalMer database}

All the results of the GalMer simulations are accessible online using Virtual Observatory technologies. The three essential blocks for providing online access to the data are: (1) storing the data; (2) storing and querying the data description, i.e. metadata; (3) mechanisms for accessing and visualising the data. We provide additional services to perform online data analysis, which are described in detail in the next section.

\subsection{Data format and storage}

Aimed at interoperability and performance, we chose the FITS binary table format (Cotton et al. 1995; Hanisch et al. 2001) to store the simulation results. The FITS format is handled by a variety of tools widely used by the astronomical community. A FITS binary table can be easily incorporated into the VOTable format (Ochsenbein et al. 2004) used by new generation astronomical software tools introduced by the Virtual Observatory.

Every galaxy interaction ("GalMer experiment" hereafter) includes 50 to 70 snapshots with a 50 Myr time interval containing data for individual particles traced by the simulations, thus following the evolution of an interacting galaxy pair for 2.5-3.5 Gyr. We store each snapshot as an individual file. The following properties are provided for every particle: cartesian coordinates $(X, Y, Z)$, three-dimensional velocity vector $\left(v_{X}, v_{Y}\right.$, $v_{Z}$ ), total mass $(M)$, particle type (hybrid, star or dark matter), identification of a galaxy to which a given particle belonged in the initial step of the simulation. In addition, we provide metallicities and $t_{\text {birth }}$, i.e. the average birth time of the stellar material in a given particle; for stellar particles, both parameters remain fixed throughout the simulations, since these particles do not evolve. For hybrid particles, we indicate instead the mean $t_{\text {birth }}$ and metallicities $z_{m}$, as well as both the gas masses and metallicities $z_{\text {new }}$ of stars formed during the previous timestep (i.e. current gas metallicity).

All the information related to the input parameters of a given GalMer experiment, i.e. morphological types of galaxies, orbital configuration, units of masses, coordinates, and velocities, as well as the epoch of a current snapshot, are provided in FITS headers of snapshot files, making them self-consistent and available for further stand-alone analysis without any need to connect to the GalMer database. 
A typical snapshot containing 240000 particles has a size of 12 MBytes, resulting in 0.6-0.9 GBytes per GalMer experiment. The present data release contains simulations of about a thousand giant-giant interactions having a total volume of $\sim 0.9$ TBytes. New simulations will be ingested into the database and put online as soon as they have been completed.

\subsection{Simulation metadata}

The metadata are computed for every individual snapshot at the time of the database update. Since the data are archived and do not change with time, neither do the metadata. The access to the database is read-only, unless new simulation results are ingested into it.

The metadata of GalMer simulations conform to the current version of the SimDB data model (Lemson et al., in prep.) being developed by the International Virtual Observatory Alliance (IVOA). SimDB is supposed to provide a complete selfsufficient description of $N$-body simulations results using objectoriented approach and is designed using the Unified Modeling Language (UML). For practical usage, such as constructing a database containing the numerical simulation metadata, a UML data model has to be serialized. We partly serialize the SimDB data model into a relational database schema keeping another part (modified Characterisation class of SimDB) serialized into native XML.

We use the open-source object-relational database management system (DBMS) PostgreSQL ${ }^{2}$ to implement the advanced metadata querying mechanisms described in detail in Zolotukhin et al. (2007). We modified the original SimDB data model by replacing its Characterisation object with the full IVOA Characterisation Data Model (CharDM) metadata (Louys et al. 2008). CharDM is a way to say where, how extended, and in which way the observational or simulated dataset can be described in a multidimensional parameter space. Our metadata querying approach allows us to use additional WHERE clauses in standard SQL queries expressed as XPath statements to constrain particular CharDM elements without any need to serialize rather complex CharDM structure into a relational database schema, and it therefore prevents additional complications of SQL queries.

The relational DBMS stores metadata including links to FITS files containing all coordinates and data of individual simulation particles. Therefore, to execute operations involving particles, such as cutouts or statistics, it is necessary to obtain the actual datasets. However, all global properties of simulations, such as the total masses of gas and stars, are available inside the DBMS making it possible to extract a global star formation history of a given GalMer experiment at the database level. This function is implemented as a stored procedure inside the SQL database and is accessible from the web interface.

All actual metadata queries are executed by the server-side of the database web interface using the parameters visualised in web pages, therefore the user does not need to type in SQL queries inside the web based forms.

\subsection{Data access and visualisation}

The interactive access to the data is provided by means of a WWW interface ${ }^{3}$. It is based on a de facto standard asynchronous JavaScript and XML (AJAX) technology and,

\footnotetext{
2 http://www.postgresql.org/

3 http://galmer.obspm.fr/
}

thus, supports most widely-used modern Internet-browsers, e.g. Mozilla Firefox ver.>1.5, Microsoft Internet Explorer ver. $>5$, and Apple Safari ver. $>3$. Individual snapshots for every simulation can also be directly accessed in a batch mode.

The web site provides a database query interface for accessing simulations of given galaxy morphological types and orbital parameters of interactions. Being a part of SimDB metadata, this information is stored in the database, and is retrieved dynamically and displayed in a pop-up window, once the user has selected particular elements in the query form. To use all available features of the GalMer database web interface, the following software components have to be installed/enabled on the user's computer: (1) JavaScript support in a web browser; (2) SUN $\mathrm{Java}^{4}$ including the Java Applet browser plug-in and support for the Java WebStart functionality, which are normally configured automatically during the installation of SUN Java.

The interactive data access involves several steps (see Fig. 4). First, galaxy morphological types and the orbital configurations of an interaction are chosen using the "DB Query" tab. Then, the user is asked to select one GalMer experiment in the "Query Results" tab from a list of those matching the selection criteria provided at the first step. Once an experiment has been selected, the user can download or visualise its integrated star formation history and/or download individual snapshots provided in the "Experiment" tab. It is then possible to preview the contents of a given snapshot and gain access to value-added data analysis tools using the "Shapshot" tab.

In the "Snapshot" tab we provide a powerful AJAX-based preview mechanism for interactive data manipulation from directly within the web browser (see bottom right panel in Fig. 4). The main purpose of the snapshot visualisation and manipulation is an interactive choice of the projection and scaling parameters for the generation of projected maps and synthetic images and/or spectra as described in the next section.

Although the interactive data visualisation capabilities available inside the web browser remain limited, this limitation can be surpassed by using dedicated software tools if the data are sent to them directly from the database web interface.

We implemented the interaction between the GalMer database web interface and existing Virtual Observatory tools dedicated to dealing with tables (topcat ${ }^{5}$, Taylor 2005), images (CDS Aladin 6 , Bonnarel et al. 2000), and spectra (ESA VOSpec $^{7}$, Osuna et al. 2008). The mechanism of interaction was proposed by Chilingarian \& Zolotukhin (2008) and initially implemented for the observational data archive ASPID-SR (Chilingarian et al. 2007). It is based on the middleware (Zolotukhin \& Chilingarian 2008) represented by two components (Fig. 5) interacting using Sun Java LiveConnect: (1) a part handling user's actions inside the web browser implemented in JavaScript; and (2) a Java applet for sending data to the VO applications, implementing a VO application messaging protocol. The present version of the middleware implements the PLASTIC (PLatform for AStronomical Tool InterConnection, Boch et al. 2006) protocol. Support for the more advanced SAMP (Simple Application Messaging Protocol, Taylor et al. 2009) will be added in the next release of the database.

\footnotetext{
4 http://www. java.com/

5 http://www.star.bris.ac.uk/\$\sim\$mbt/topcat/

6 http://aladin.u-strasbg.fr/

7 http://esavo.esa.int/vospec/
} 

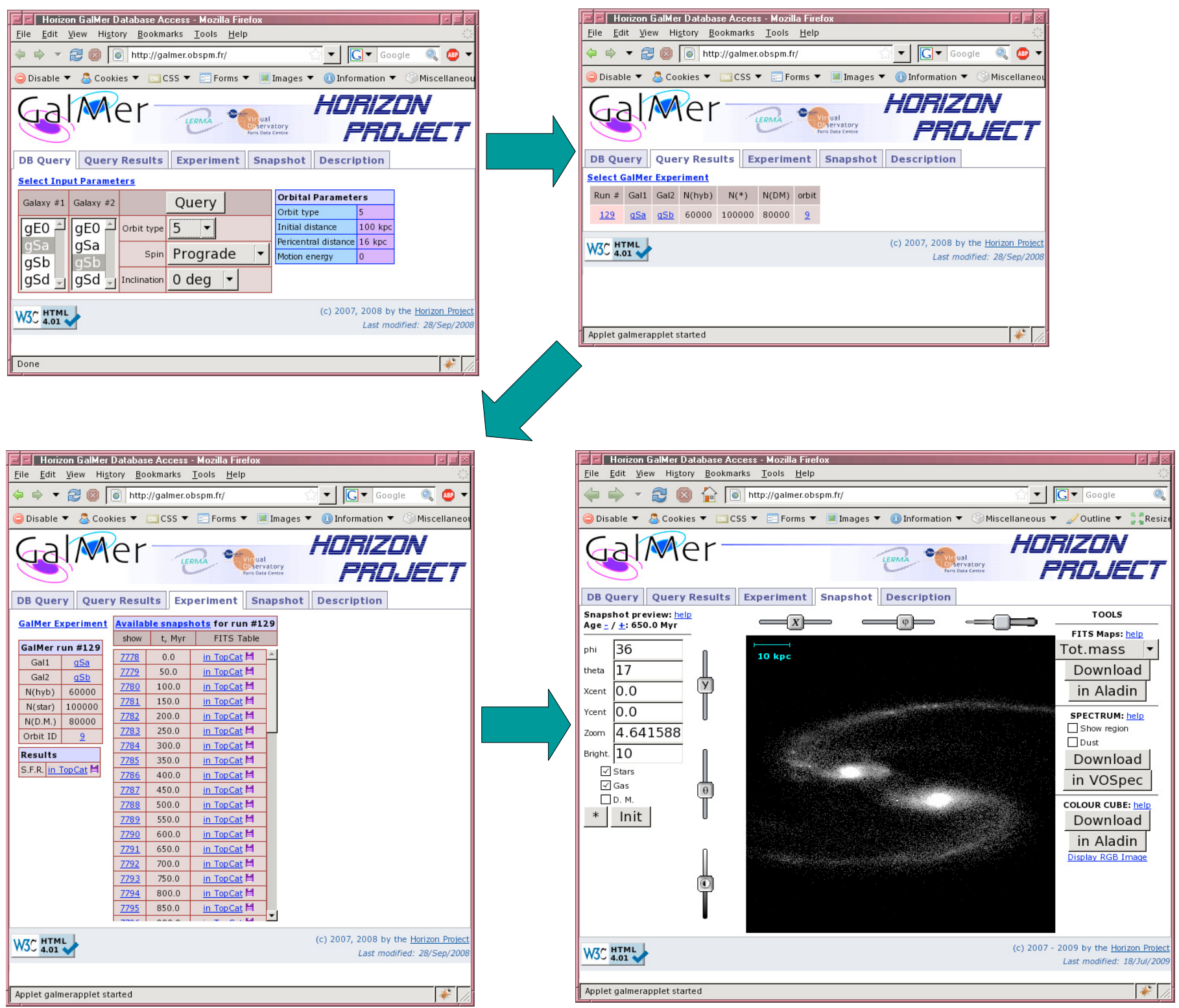

Fig. 4. Interactive data access using the GalMer web interface: (1) selecting galaxy morphologies and orbital types; (2) choosing one GalMer experiment from the list of those matching the selection criteria; (3) selecting a snapshot; (4) previewing the snapshot and accessing data analysis tools for it.

\section{Data analysis services}

We developed an application programming interface (API) library to access and analyse the results of GalMer simulations. The mergerapi library is implemented in ANSI C and needs a minimal set of prerequisites to compile: the cfitsio FITS API library $^{8}$ to access the simulation data and the GD library ${ }^{9}$ to generate gif and png output images directly viewable inside a web browser. The mergerapi library is installed on the server side and is used by several on-the-fly data analysis services described hereafter.

\subsection{Projected maps}

For a given snapshot, we provide a set of services for the onthe-fly server-side computation of projected maps of various

\footnotetext{
8 http://heasarc.gsfc.nasa.gov/docs/software/fitsio/

fitsio.html

9 http://www.libgd.org/
}

quantities traced by the simulations. These tools are accessible at the "Snapshot" tab at the GalMer web site.

The following quantities can be computed for any combination of the three particle types: surface density, line-of-sight radial velocity $\left(v_{r}\right)$, and line-of-sight velocity dispersion $(\sigma)$. The gas mass, stellar mass, gas metallicity, and metallicity of new stars are provided for hybrid particles only. Stellar metallicity represents the metallicity of the underlying ("old") stellar population, therefore it applies to "star" particles and remains constant in time for a given particle. Preview surface density maps are shown directly in a web browser, while for other quantities (velocities, metallicities, etc.) the maps are available for download as FITS images or can be visualised with CDS Aladin.

To generate maps on-the-fly, we developed an efficient computational algorithm. All the maps are computed for a parallel projection onto a plane (i.e. assuming an observer at the infinite distance), at the same time allowing us to specify a spatial scale (i.e. to derive an effective distance from an observer to the barycentre $r$ ). Therefore, the viewport is uniquely defined by the 

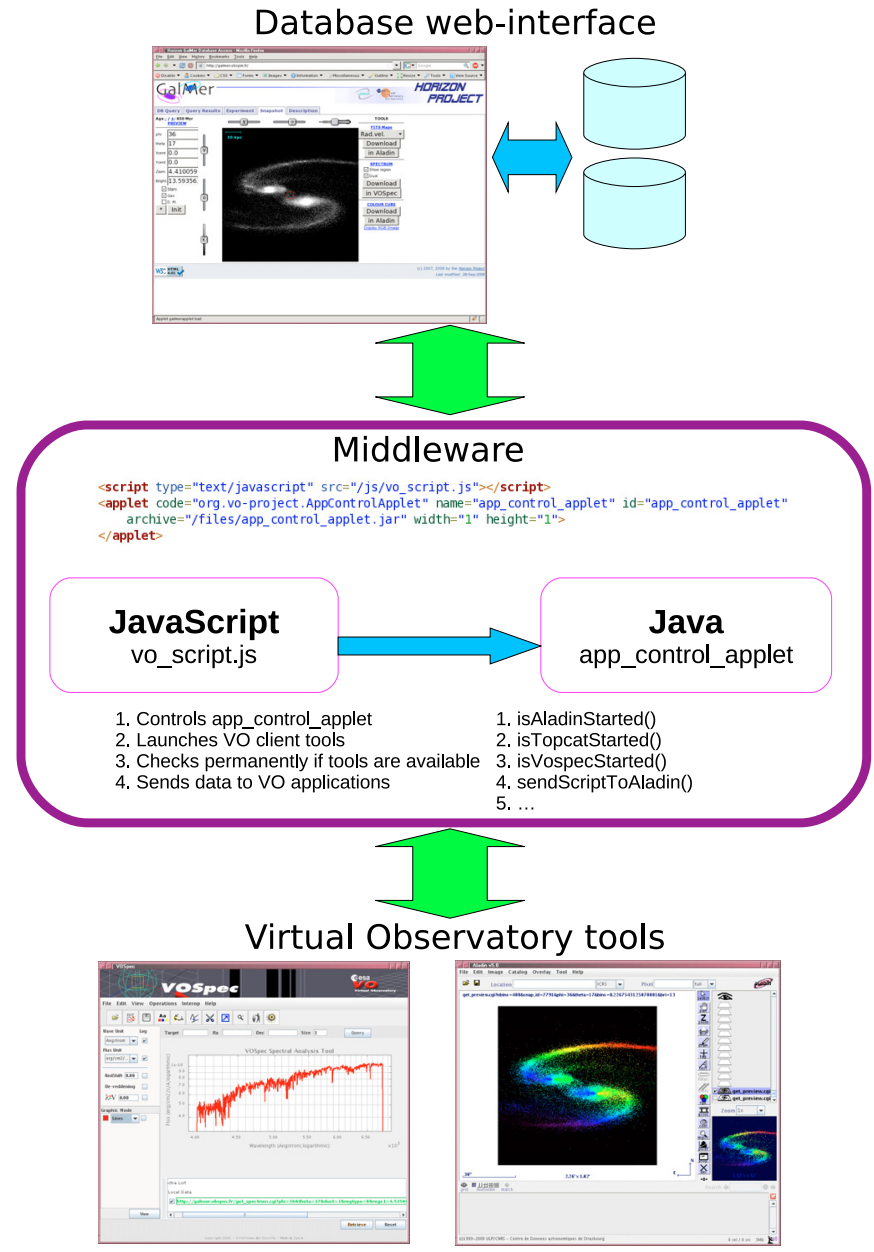

Fig. 5. The middleware connecting the GalMer database web interface to the $\mathrm{VO}$ tools dedicated to advanced data manipulation is illustrated at top, as a projection with mergerapi, and at bottom, as both a VOSPEC spectrum and a map in Aladin.

two quantities: azimuthal $(\varphi)$ and polar $(\theta)$ angles. To simplify the comparison of both the computed maps with observations and the mosaicking of maps with different spatial sampling (e.g. zoom-in on certain regions of a merger remnant with the overall image), we use the FITS WCS convention (Greisen \& Calabretta 2002) assuming a tangential projection and assigning right ascension and declination of zero to the projected barycentre position. In this case, the coordinates of a particle on the projected plane are computed to be:

$$
\begin{aligned}
x_{\text {proj }} & =-X \sin \varphi+Y \cos \varphi \\
\eta & =206265 x_{\text {proj }} / r \\
y_{\text {proj }} & =-X \cos \varphi \sin \theta-Y \sin \varphi \sin \theta+Z \cos \theta \\
\xi & =206265 y_{\text {proj }} / r .
\end{aligned}
$$

Here $\eta$ and $\xi$ define the tangential coordinates on the sky in arcsec used to produce synthetic images directly comparable to observations as demonstrated in Sect. 6.2.

The radial velocity and the position on the line of sight are given by:

$v_{\mathrm{r}}=v_{X} \cos \varphi \cos \theta+v_{Y} \sin \varphi \cos \theta+v_{Z} \sin \theta$

$z_{\mathrm{r}}=X \cos \varphi \cos \theta+Y \sin \varphi \cos \theta+Z \sin \theta$.

We then perform the following steps:

- A grid corresponding to the desired map size and sampling is created.
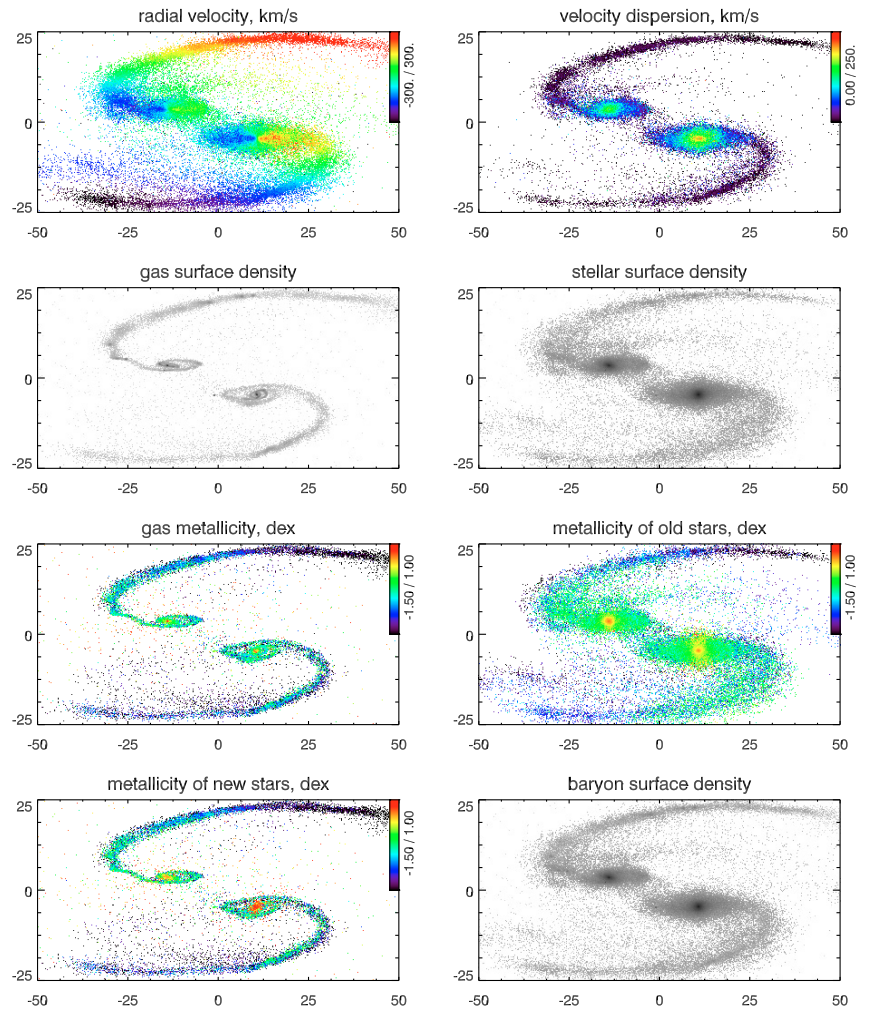

Fig. 6. Projected maps of 8 quantities traced by the GalMer simulations for a merger of gSa and $\mathrm{gSb}$, prograde orbit of type 5, $650 \mathrm{Myr}$ after the start of the simulations. Left-to-right, top-to-bottom: radial velocity, velocity dispersion (star and hybrid particles), projected density of gas, projected density of old stars, metallicity of gas, metallicity of old stars, metallicity of new stars, and total projected density (star + hybrid particles).

- In a single loop over all particles, we determine the bin to which each particle will contribute, and take account of this contribution.

- In a second loop over the bins (pixels), we compute (if necessary) the actual values of a physical parameter.

The algorithm is sufficiently fast to perform the map computation in real time, e.g. the generation of a $400 \times 400$ pixels map takes a fraction of a second. In Fig. 6, we show an example of projected maps for one of the interactions.

\subsection{Spectrophotometric properties}

We developed a technique using PEGASE.HR (Le Borgne et al. 2004) and PEGASE.2 (Fioc \& Rocca-Volmerange 1997) to model spectrophotometric properties of interacting galaxies from the results of GalMer simulations. By taking into account kinematics, star formation (SFH), and chemical enrichment history (CEH), we model spectra and broadband photometric colours. We will present all the details regarding the spectrophotometric modelling in a separate paper (Chilingarian et al., in prep.), but here we briefly describe the algorithms and results that can be obtained.

There were several successful attempts (Chakrabarti \& Whitney 2009; Jonsson et al. 2010) to model the spectrophotometric properties of the results of $N$-body simulations. However, in all the known cases the stellar population information is taken into account in an approximate way, by characterising $N$-body particles by their mean ages and metallicities. At the same time, 
the behaviour of spectral features is a strongly non-linear function of age and metallicity and it also differs significantly across the wavelength domain, in particular, bcause of the dust attenuation. Hence, SEDs computed using mass-weighted average ages and metallicities may not reflect the real spectral energy distributions of galaxies. With the GalMer simulations, thanks to the usage of hybrid particles we are (1) able to trace SFH and $\mathrm{CEH}$ in detail through the entire duration of the simulation for every particle of this type; and (2) make use of this information. Therefore, we are able to perform qualitatively superior modelling of the spectrophotometric properties of interacting galaxies (and results of other $N$-body TreeSPH simulations as well) and make direct comparison with observations. The SFH and CEH are computed using a whole sequence of snapshots in a given GalMer experiment, as differences in gas masses of each hybrid particle in each of the 10 metallicity bins from $[\mathrm{Fe} / \mathrm{H}]=-2.5$ to $+1.0 \mathrm{dex}$.

The most time-consuming part of the modelling is running the PEGASE.HR code. Because of the complexity of the evolutionary synthesis even with the present state-of-the-art computer hardware, it takes several seconds per spectrum (i.e. per star particle), resulting in several hours per snapshot and including up to 160000 hybrid and star particles. Computation of spectra for individual particles is absolutely crucial to properly model the effects of the dust extinction and intrinsic broadening of spectral lines caused by motions of particles along a line of sight (i.e. internal kinematics of galaxies). However, we can avoid executing of evolutionary synthesis code.

The algorithms of evolutionary synthesis such as PEGASE.HR include:

1. computation of isochrones for different ages and metallicities based on a given set of stellar evolutionary tracks and stellar IMF;

2. picking up stellar spectra from a stellar library (either empirical or theoretical) for the atmosphere parameters corresponding to a given point on the isochrone;

3. coadding contributions of different types of stars according to the weights on the isochrone, to produce a simple stellar population (SSP) spectrum including stars of a single age and metallicity;

4. coadding different SSPs to reproduce complex SFH and $\mathrm{CEH}$.

When the IMF is fixed and both the SFH and CEH are traced on a pre-defined grid of ages and metallicities, it becomes possible to execute only the 4th step from the list above. This means that if we pre-compute only once a grid of SSPs corresponding to a given IMF (Miller \& Scalo 1979 in our case) and the grid of ages and metallicities, we can reuse it for all the particles, improving the efficiency of the computations by several orders of magnitude. Once for every GalMer experiment, we also precompute the SFH and $\mathrm{CEH}$ and store them as a two-dimensional histogram for every particle.

For every spatial bin (see definition in the previous section), we first sort the particles along a line of sight to account for dust extinction. Then, either a high-resolution spectrum or a broadband spectral energy distribution (depending on the mode of the computation) is computed for every particle starting from the most distant one from the observer. This is performed by coadding the pre-computed SSPs from the grid mentioned above with the weights corresponding to a mass contribution of stars of each age and metallicity contained in the SFH and CEH.

The dust extinction is then applied to the total spectrum or multi-colour spectral energy distribution (SED) computed at the

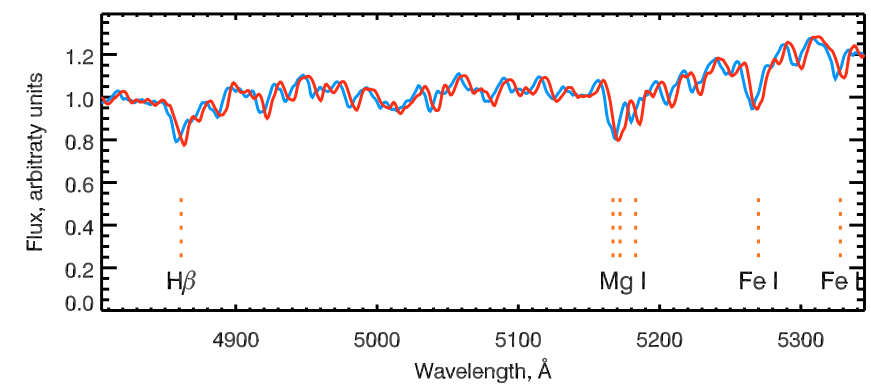

Fig. 7. Examples of synthetic spectra generated by the spectrophotometric modelling algorithm applied to the results of GalMer simulations. Two curves correspond to two different positions along the projected major axis of a merger remnant: centre approaching (blue), and receding (red). The spectra are normalised to unity at $\lambda=5000 \AA$ for clarity.

previous step (i.e. excluding the current particle). For a known gas column density and a solar metallicity, we assume a standard dust-to-gas mass ratio to compute extinction as $A_{V}=N_{\mathrm{H}} / 1.871 \times$ $10^{21}$ (Bohlin et al. 1978), where $N_{\mathrm{H}}$ is a number of hydrogen atoms per $\mathrm{cm}^{2}$, assuming $R_{V} \equiv A_{V} / E(B-V)=3.1$. We compute $N_{\mathrm{H}}$ along the line of sight and scale this formula linearly with metallicity. We then account for the wavelength dependence of extinction according to Fitzpatrick (1999), and apply it to the total stellar population SED generated at this step.

After having applied the dust extinction, the total spectrum of a current particle is blue- or redshifted according to its radial velocity, by performing a simple shift operation with linear interpolation, since our SSP grid is rebinned with a logarithmic step in the wavelength corresponding to a fixed pixel size in $\mathrm{km} \mathrm{s}^{-1}$.

The total spectrum (or SED) of a current particle is then coadded to the result. For a given snapshot, this algorithm makes it possible to compute in a few seconds a total intermediateresolution $(R=3000,3900<\lambda<6800 \AA)$ spectrum, based on the ELODIE.3.1 (Prugniel et al. 2007) empirical stellar library. The total number of coadded SSP in this extreme case reaches $10^{6}$. The broad-band FUV to NIR SED based on the lowresolution theoretical BaSeL (Lejeune et al. 1997) stellar library is computed much faster, because the number of SED points in it is only about a dozen compared to several thousands, allowing us to compute a $400 \times 400$ pixels multi-colour datacube in a few seconds.

Because of the limited spatial resolution of GalMer simulations, we are unable to reach the spatial scales sufficient to take into account properly the gas clumpiness, therefore we adopt a scale of $250 \mathrm{pc}$ for the computation of extinction, which corresponds roughly to the resolution of the simulations, and produces the values of total extinction in simulated GalMer isolated galaxies that closely resemble observations. Our limited resolution also causes overestimation of extinction effects in the central regions with high gas densities, where large amounts of gas falls during the interaction. We do not presently include any dust emission in mid- and far-IR spectral bands. This will be performed in a future release of the GalMer database and presented in a separate paper (Melchior et al. in prep.)

In Fig. 7, we provide an example of the spectra generated for merger remnants, where the effects of the internal kinematics are clearly visible. Red and blue spectra taken $1.5 \mathrm{kpc}$ from the galaxy centre along the projected major axis, demonstrate the Doppler shift of spectral lines. Absorption lines are thus broadened naturally and reflect the intrinsic stellar velocity dispersion of the galaxy. 
At present, we do not attempt to model nebular emission lines, because our simulations do not trace at a sufficient level of detail the physical conditions in the ISM. Only qualitative modelling can in principle be performed, assuming a fixed ISM temperature and density depending only on the gas metallicity available for hybrid particles. This modelling will be addressed in detail in a separate paper (Melchior et al., in prep.)

\section{Astrophysical applications}

\subsection{Galaxy properties from modelling}

We have explored the GalMer database so far to study a variety of physical processes related to galaxy interactions, such as induced star formation enhancements, evolution of metallicity gradients, angular momentum redistribution, and their impacts on the final kinematical properties of the merger remnant.

In Di Matteo et al. (2007) and Di Matteo et al. (2008a), we investigated the enhancement of star formation efficiency in galaxy interactions and mergers. In Di Matteo et al. (2007) we showed that, (i) in the final merging phase, retrograde encounters have greater star formation efficiency than direct encounters; (ii) that the amount of gas available in the galaxy is not the main parameter governing the star formation efficiency during the burst phase; and (iii) that there is a negative correlation between the amplitude of the star forming burst at the merging phase and the tidal forces exerted at pericentral passage. The general result presented in Di Matteo et al. (2008a) shows that, at low redshift, galaxy interactions and mergers, in general, trigger only moderate star formation enhancements. Strong starbursts where the star formation rate is increased by a factor greater than 5 are rare and found only in about $15 \%$ of major galaxy interactions and mergers. Merger-driven starbursts are also rather shortlived, with a typical duration of activity of a few $10^{8} \mathrm{yr}$. These conclusions are found to be robust, independent of the numerical techniques and star formation models. At higher redshifts, where galaxies are gas-rich, gas-inflow-induced starbursts are neither stronger nor longer than their local counterparts. These results are in good agreement with a number of observational works (Bergvall et al. 2003; Li et al. 2008; Jogee et al. 2009; Knapen \& James 2009), demonstrating that interactions and mergers do not always trigger strong bursts of star formation.

Di Matteo et al. (2009b) investigated how the metallicity gradients in dry merger remnants depend on the structure and metallicity gradients of the galaxies involved in the merger. Our aim was to understand whether dry mergers could produce the metallicity gradients observed in elliptical galaxies in the local Universe, and always cause a flattening of the initial (i.e., premerger) gradient. The analysis of the entire set of dry merger simulations in the GalMer database allowed us to demonstrate that the ratio of the remnant to the initial galaxy slopes spans a wide range of values, up to $>1$ (with values greater than one resulting only when companions have gradients twice that of the progenitor). For a merger between two ellipticals with identical initial metallicity slopes (i.e., equal companion and galaxy slopes), the metallicity profile of the remnant flattens, the final gradient being about 0.6 times the initial one. This flattening depends neither on the characteristics of the orbit of the progenitors nor on their initial concentration. If the companion slope is sufficiently steep, ellipticals can maintain their original pre-merger metallicity gradient. When compared to the observed variety of metallicity gradients in dwarf elliptical and lenticular galaxies (Chilingarian 2009), these results infer that mergers may be an important process in $\mathrm{dE} / \mathrm{dS} 0$ evolution. Given the diversity in the outcomes of the mergers, we concluded that dry mergers do not violate any observational constraints provided by the typically measured metallicity gradients in local ellipticals (Ogando et al. 2005). The redistribution of the orbital angular momentum into internal rotation, and its impact on the kinematical properties of the merger remnant, were the subject of two separate studies (Di Matteo et al. 2008b, 2009a).

In Di Matteo et al. (2008b), we presented a new scenario to form counter-rotating central components in early-type galaxies, by means of dissipative and dissipationless "mixed" mergers, consisting of elliptical-spiral systems in retrograde orbits. We demonstrated that the counter-rotation can appear in both dissipative and dissipationless retrograde mergers and is mostly associated with the presence of a disc component, which preserves part of its initial spin. In turn, the external regions of the two interacting galaxies acquire part of the orbital angular momentum, due to the action of tidal forces exerted on each galaxy by the companion. In the case of dissipative mergers, the central decoupled core may consist of two distinct populations: the old stellar population, which has preserved part of its initial spin, and a new stellar population, born in situ from the kinematically decoupled gas component.

Even the merger of two initially non-rotating, pressuresupported progenitor galaxies can lead to remnant galaxies with peculiar kinematical properties. In Di Matteo et al. (2009a), we demonstrated that it is possible to generate elliptical-like galaxies, with $v / \sigma>1$ outside one effective radius, by converting orbital into internal angular momentum. This conversion occurs "outside-in", the external regions acquiring part of the angular momentum first, then affecting both the baryonic and the dark matter components of the remnant galaxy (i.e. both acquire part of the angular momentum, the relative fractions depend on the initial concentration of the merging). If the initial baryonic component is sufficiently dense and/or the encounter takes place on a orbit with high angular momentum, the remnant galaxy exhibits hybrid properties, i.e. an elliptical-like morphology, but rotational support in the outer stellar halo $(v / \sigma>1)$. Systems with these properties have been observed by means of a combination of stellar absorption lines and planetary nebulae for kinematic studies of early-type galaxies (Coccato et al. 2009). Our results are in qualitative agreement with these observations and demonstrate that even mergers composed of non-rotating, pressure-supported progenitor galaxies can produce early-type galaxies with significant rotation at large radii.

\subsection{Synthetic observations: virtual telescope}

Some of the value-added data analysis services that we provide for the GalMer database can be used as a "virtual telescope" to create simulated images and spectra of interacting galaxies. Thanks to the high-quality stellar population modelling provided by the PEGASE.2/PEGASE.HR code, we are able to compare the simulated data to real observations.

In Fig. 8, we present an RGB false-colour composite image of an interacting galaxy pair created from the results of spectrophotometric modelling described above applied to GalMer simulations. The simulation result is superimposed on a background of the Sloan Digital Sky Survey Data Release 7 (Abazajian et al. 2009) image of the galaxy cluster Abell 85. We used SDSS $g, r$, and $i$ bands in both observational and simulated data, and the RGB visualisation code implementing the algorithm by Lupton et al. (2004) to generate a false-colour image. Since the results of our spectrophotometric modelling are expressed in physical units, they can be transformed into 


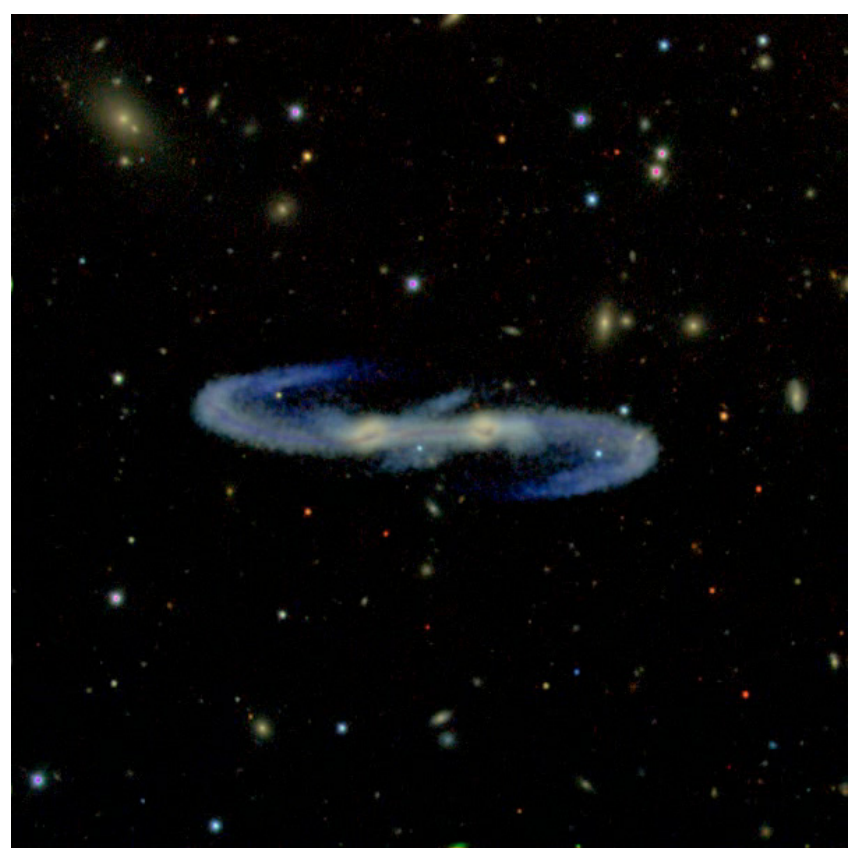

Fig. 8. RGB false-colour image of a simulated galaxy merger displayed over the SDSS image of the Abell 85 galaxy cluster.

observables (mag $\operatorname{arcsec}^{-2}$ ) and projected onto the sky (see Eq. (6)), providing a direct way of comparing them to observational data. By coadding model broadband fluxes and those derived from SDSS direct imaging, we can create realistical falsecolour images such as the one shown in Fig. 8.

Simulated images can then be analysed using classical observational techniques, such as surface photometry. A successful example of this analysis is demonstrated in Chilingarian et al. (2009b), where we found a match between a rather complex three-component observed density profile of the lenticular galaxy NGC 6340 and a merger remnant from the GalMer simulations, supporting the major merger to be an important event in the evolution of this particular object.

We are also able to simulate the whole data cubes corresponding to the data produced by modern integral-field unit (IFU) spectrographs, such as SAURON at $4.2 \mathrm{~m}$ William Herschel Telescope, VIMOS at ESO Very Large Telescope, and GMOS at Gemini. Our spectral resolution and coverage allows us to model high-resolution blue setting of VIMOS (HR-Blue, $R=2500)$ and B600 grating of GMOS $(R=2200)$ usually chosen to study stellar kinematics of nearby galaxies.

The spatial resolution of our simulations $(0.20-0.28 \mathrm{kpc})$ is comparable to that of the SAURON (de Zeeuw et al. 2002) survey targeting nearby galaxies at distances between 10 and $40 \mathrm{Mpc}$, thus, having spatial resolution of 0.05 to $0.2 \mathrm{kpc}$ given the average atmosphere seeing quality of 1 arcsec. At the same time, we can degrade the spatial resolution of our simulation to match observations made with VIMOS, e.g. post-starburst galaxies presented in Chilingarian et al. (2009a).

As the stellar population models improve, we will be able to upgrade the spectrophotometric modelling engine in the GalMer database to simulate data from next generation IFU facilities such as the second-generation VLT instrument MUSE.

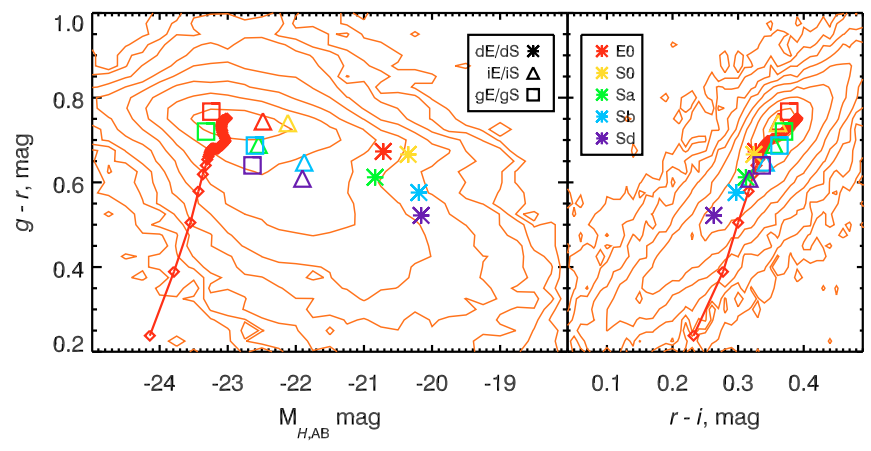

Fig. 9. Colour-magnitude (left) and colour-colour relations demonstrating contour density plots for 80000 observed low-redshift $(z<0.3)$ SDSS galaxies, GalMer galaxy models corresponding to isolated galaxies of different masses and morphological types, and a sequence of a GalMer simulation of a giant-giant interaction followed from the merger epoch for $1.5 \mathrm{Gyr}$ every $50 \mathrm{Myr}$ (red diamonds). Colours (see right panel legend) code morphological type, while symbol shapes (asterisk, triangle and square, see left panel legend) code luminosity-class.

\subsection{Colour-magnitude relations}

The spectrophotometric modelling facilities allow us to compute total magnitudes and colour of interacting galaxies and to directly compare them to observational data.

The colour-magnitude and colour-colour plots comparing results of GalMer simulations to a large observational dataset are presented in Fig. 9. A well-known galaxy colour bimodality (see e.g. Strateva et al. 2001) is clearly seen in the contour plot presenting the distribution of some 80000 nearby galaxies $(z<0.3)$ from the SDSS survey. The photometric data were corrected for Galactic extinction according to Schlegel et al. (1998) and converted into rest-frame, i.e. $k$-corrected using the analytical approximations presented in Chilingarian et al. (2010). The "red sequence" and the "blue cloud" separated by the "green valley", a locus of post-starburst galaxies (e.g. Goto et al. 2003) can be identified.

The total magnitudes of GalMer mergers in SDSS bands are computed using the spectrophotometric modelling described above. We show the positions of GalMer galaxy models (isolated galaxies) of different masses and morphological types. Elliptical and lenticular galaxies (red and yellow symbols) are sitting on the red sequence, while spiral galaxies are bluer, directed down toward the blue cloud. GalMer Sa and Sb spiral galaxies (green and blue symbols) at an evolutionary stage shown in Fig. 9 reside in the green valley.

We also follow the evolution of an interacting pair from the time when galaxies merged (red diamonds connected with lines) until the end of the simulation showing intermediate snapshots every 50 Myr. Immediately after the gas-rich merger, remnants sit in the "blue cloud", despite very strong internal extinction of regions of massive star formation. Then, as the star formation decreases, luminosity-weighted ages of stellar populations increase causing global colours to become redder and, thus, moving merger remnants in the top-right direction across the "blue cloud" through the "green valley" toward the "red sequence". Finally, 400-600 Myr after the merger time, all remnants settle onto the "red sequence", although some residual star formation is still observed.

One can see that merger remnants cross the "green valley" very rapidly, possibly explaining why there is at present a deficit of galaxies in this region of the colour-magnitude diagram, given that all mergers in this mass range have not yet been included in the database. 


\section{Summary}

We have presented the GalMer database providing online access to the results of TreeSPH simulations. The structure of the database conforms to International Virtual Observatory standards. We describe the interactive data access web interface, and advanced mechanisms for data visualisation and manipulation using connection to the Virtual Observatory tools dedicated for dealing with tabular, imaging and spectral data.

After having stored the snapshots in FITS binary table format, giving access to all coordinates and data on individual simulation particles, we provide a set of value-added tools using the results of TreeSPH simulations. These include: (1) a generator of projected maps of various physical quantities traced by the simulations; (2) an engine to perform the modelling of spectrophotometric properties of interacting and merging galaxies based on the PEGASE.2/PEGASE.HR evolutionary synthesis code. The latter tool can be used as a virtual telescope and synthetic images, spectra, and datacubes generated using it are directly comparable to observational data. We provide examples of several applications for the GalMer database using our valueadded tools.

The database will be updated by including new simulations when they are completed.

Acknowledgements. This research used computational resources of the Informatic Division of the Paris Observatory, Commissariat à l'Énergie Atomique, the CNRS national centre IDRIS, and those available within the framework of the Horizon project. A server dedicated to the on-the-fly computations and the database in Paris Observatory has been provided by the VO Paris Data Centre. I.C. acknowledges additional support from the RFBR grant 07-0200229-a.

Funding for the SDSS and SDSS-II has been provided by the Alfred P. Sloan Foundation, the Participating Institutions, the National Science Foundation, the U.S. Department of Energy, the National Aeronautics and Space Administration, the Japanese Monbukagakusho, the Max Planck Society, and the Higher Education Funding Council for England. The SDSS Web Site is ${ }^{10}$.

\section{Appendix A: Galaxy models: density profiles}

In Sect. 2.1, we presented the galaxy models adopted for the simulations. In particular, we saw that the dark halo and the optional bulge are modelled as a Plummer sphere (Binney \& Tremaine 1987, pag. 42), with characteristic masses $M_{\mathrm{B}}$ and $M_{\mathrm{H}}$ and characteristic radii $r_{\mathrm{B}}$ and $r_{\mathrm{H}}$. Their densities are given, respectively, by the following analytical formula:

$\rho_{\mathrm{H}}(r)=\left(\frac{3 M_{\mathrm{H}}}{4 \pi r_{\mathrm{H}}^{3}}\right)\left(1+\frac{r^{2}}{r_{\mathrm{H}}^{2}}\right)^{-5 / 2}$

and

$\rho_{\mathrm{B}}(r)=\left(\frac{3 M_{\mathrm{B}}}{4 \pi r_{\mathrm{B}}^{3}}\right)\left(1+\frac{r^{2}}{r_{\mathrm{B}}^{2}}\right)^{-5 / 2}$.

The stellar and gaseous discs follow a Miyamoto-Nagai density profile (Binney \& Tremaine 1987, pag. 44):

$$
\begin{aligned}
\rho_{*}(R, z)= & \left(\frac{h_{*}^{2} M_{*}}{4 \pi}\right) \\
& \times \frac{a_{*} R^{2}+\left(a_{*}+3 \sqrt{z^{2}+h_{*}^{2}}\right)\left(a_{*}+\sqrt{z^{2}+h_{*}^{2}}\right)^{2}}{\left[a_{*}^{2}+\left(a_{*}+\sqrt{z^{2}+h_{*}^{2}}\right)^{2}\right]^{5 / 2}\left(z^{2}+h_{*}^{2}\right)^{3 / 2}}
\end{aligned}
$$

${ }_{10}$ http://www.sdss.org/

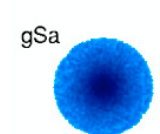

$\mathrm{gSb}$
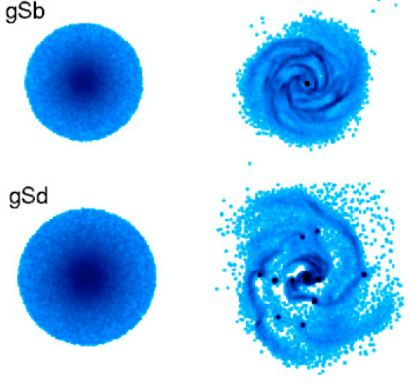
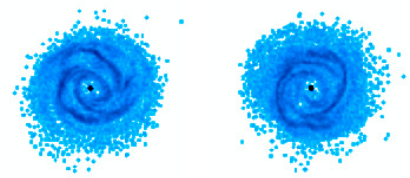

Fig. B.1. Gas maps of the gSa, gSb, and gSd models with $Q_{\text {gas }}=0.3$ evolved in isolation. Snapshots are equally spaced in time from $t=0$ to $t=3$ Gyr. Each map is $60 \mathrm{kpc} \times 60 \mathrm{kpc}$ in size.

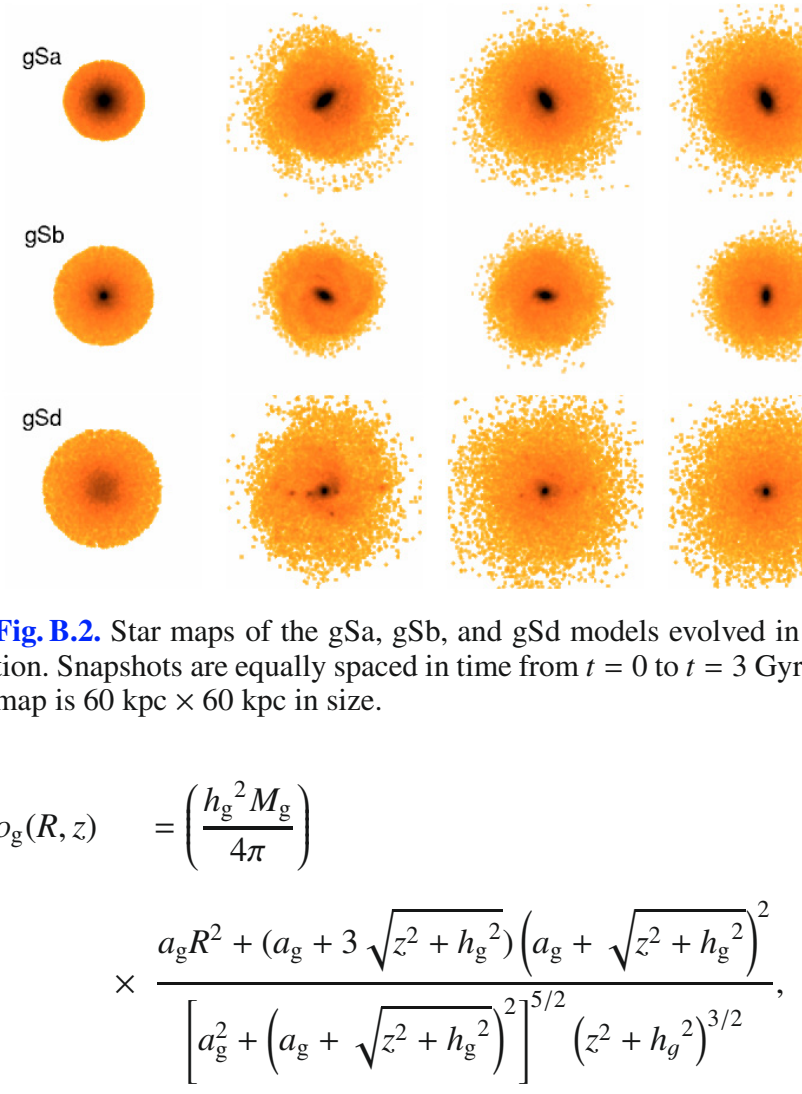

where the masses $M_{*}$ and $M_{\mathrm{g}}$ and vertical and radial scale lengths are represented respectively, by $h_{*}$ and $a_{*}$, and $h_{\mathrm{g}}$ and $a_{\mathrm{g}}$.

\section{Appendix B: Galaxy models evolved in isolation}

Since we aim to study the effects of interactions and mergers on galaxy evolution, it is also important to study, in parallel, the evolution of the galaxies in isolation, to distinguish properly secular processes from those related to the interaction. In Figs. B.1 and B.2, we show the evolution of the gaseous and stellar components of the giant $\mathrm{gSa}, \mathrm{gSb}$ and gSd galaxies, respectively, evolved in isolation for $3 \mathrm{Gyr}$. As can be seen, all the models evolve rapidly in the first 0.5-1 Gyr: a stellar bar and spiral arms form, and at the same time gas compresses into density waves and clumps and partially falls into the central regions, where a 
star formation enhancement takes place (for the determination of the star formation rate of these galaxies, we refer the reader to Di Matteo et al. 2007).

\section{References}

Abazajian, K. N., Adelman-McCarthy, J. K., Agüeros, M. A., et al. 2009, ApJS, 182,543

Barnes, J., \& Hut, P. 1986, Nature, 324, 446

Barnes, J. E., \& Hernquist, L. 1996, ApJ, 471, 115

Bergvall, N., Laurikainen, E., \& Aalto, S. 2003, A\&A, 405, 31

Binney, J., \& Tremaine, S. 1987, Galactic dynamics, ed. J. Binney, \& S. Tremaine

Blaizot, J., Guiderdoni, B., Devriendt, J. E. G., et al. 2004, MNRAS, 352, 571

Boch, T., Comparato, M., Taylor, J., Taylor, M., \& Winstanley, N. 2006, IVOA Note, version 1.00, 01 June

Bohlin, R. C., Savage, B. D., \& Drake, J. F. 1978, ApJ, 224, 132

Boissier, S., Prantzos, N., Boselli, A., \& Gavazzi, G. 2003, MNRAS, 346, 1215

Bonnarel, F., Fernique, P., Bienaymé, O., et al. 2000, A\&AS, 143, 33

Bournaud, F., Jog, C. J., \& Combes, F. 2005, A\&A, 437, 69

Bournaud, F., Jog, C. J., \& Combes, F. 2007, A\&A, 476, 1179

Bournaud, F., Duc, P.-A., \& Emsellem, E. 2008, MNRAS, 389, L8

Chakrabarti, S., \& Whitney, B. A. 2009, ApJ, 690, 1432

Chilingarian, I., Afanasiev, V., Bonnarel, F., et al. 2007, in Astronomical Data Analysis Software and Systems XVI, ed. R. A. Shaw, F. Hill, \& D. J. Bell, ASP Conf. Ser., 376, 217

Chilingarian, I., \& Zolotukhin, I. 2008, in Astronomical Data Analysis Software and Systems XVII, ed. R. W. Argyle, P. S. Bunclark, \& J. R. Lewis, ASP Conf. Ser., 394, 351

Chilingarian, I. V. 2009, MNRAS, 394, 1229

Chilingarian, I. V., De Rijcke, S., \& Buyle, P. 2009a, ApJ, 697, L111

Chilingarian, I. V., Novikova, A. P., Cayatte, V., et al. 2009b, A\&A, 504, 389

Chilingarian, I. V., Melchior, A.-L., \& Zolotukhin, I. Yu. 2010, MNRAS, 405, 1409

Coccato, L., Gerhard, O., Arnaboldi, M., et al. 2009, MNRAS, 394, 1249

Combes, F., Dupraz, C., \& Gerin, M. 1990, Gas dynamics in a tidal interaction: formation of rings., ed. R. Wielen, 205

Cotton, W. D., Tody, D., \& Pence, W. D. 1995, A\&AS, 113, 159

Cox, T. J., Jonsson, P., Primack, J. R., \& Somerville, R. S. 2006, MNRAS, 373, 1013

Cox, T. J., Jonsson, P., Somerville, R. S., Primack, J. R., \& Dekel, A. 2008, MNRAS, 384, 386

de Zeeuw, P. T., Bureau, M., Emsellem, E., et al. 2002, MNRAS, 329, 513

Di Matteo, P., Combes, F., Melchior, A.-L., \& Semelin, B. 2007, A\&A, 468, 61

Di Matteo, P., Bournaud, F., Martig, M., et al. 2008a, A\&A, 492, 31

Di Matteo, P., Combes, F., Melchior, A.-L., \& Semelin, B. 2008b, A\&A, 477, 437

Di Matteo, P., Jog, C. J., Lehnert, M. D., Combes, F., \& Semelin, B. 2009a, A\&A, 501, L9

Di Matteo, P., Pipino, A., Lehnert, M. D., Combes, F., \& Semelin, B. 2009b, A\&A, 499, 427

Dressler, A. 1980, ApJ, 236, 351

Evrard, A. E. 1988, MNRAS, 235, 911

Fioc, M., \& Rocca-Volmerange, B. 1997, A\&A, 326, 950

Fitzpatrick, E. L. 1999, PASP, 111, 63

Gao, Y., \& Solomon, P. M. 2004, ApJ, 606, 271

Gingold, R. A., \& Monaghan, J. J. 1982, J. Comp. Phys., 46, 429

Goto, T., Nichol, R. C., Okamura, S., et al. 2003, PASJ, 55, 771

Greisen, E. W., \& Calabretta, M. R. 2002, A\&A, 395, 1061

Hanisch, R. J., Farris, A., Greisen, E. W., et al. 2001, A\&A, 376, 359

Hernquist, L. 1993, ApJS, 86, 389

Hernquist, L., \& Katz, N. 1989, ApJS, 70, 419

Hitschfeld, M., Kramer, C., Schuster, K. F., Garcia-Burillo, S., \& Stutzki, J. 2009, A\&A, 495, 795

Jimenez, R., Slosar, A., Verde, L., et al. 2010, MNRAS, 404, 975

Jogee, S., Miller, S. H., Penner, K., et al. 2009, ApJ, 697, 1971

Jonsson, P., Groves, B. A., \& Cox, T. J. 2010, MNRAS, 403, 17

Jungwiert, B., Combes, F., \& Palouš, J. 2001, A\&A, 376, 85
Katz, N. 1992, ApJ, 391, 502

Kennicutt, R. C., Calzetti, D., Walter, F., Prescott, M. K., \& SINGS Team 2005, in Bulletin of the American Astronomical Society BAAS, 37, 1248

Kennicutt, Jr., R. C. 1998, ARA\&A, 36, 189

Kennicutt, Jr., R. C., Bresolin, F., \& Garnett, D. R. 2003, ApJ, 591, 801

Knapen, J. H., \& James, P. A. 2009, ApJ, 698, 1437

Kuntschner, H., Emsellem, E., Bacon, R., et al. 2006, MNRAS, 369, 497

Le Borgne, D., Rocca-Volmerange, B., Prugniel, P., et al. 2004, A\&A, 425, 881

Lee, H., Skillman, E. D., Cannon, J. M., et al. 2006, ApJ, 647, 970

Lejeune, T., Cuisinier, F., \& Buser, R. 1997, A\&AS, 125, 229

Lemasle, B., François, P., Piersimoni, A., et al. 2008, A\&A, 490, 613

Li, C., Kauffmann, G., Heckman, T. M., Jing, Y. P., \& White, S. D. M. 2008, MNRAS, 385, 1903

Louys, M., Richards, A., Bonnarel, F., et al. 2008, IVOA Recommendation, version 1.13, 25 March

Lucy, L. B. 1977, AJ, 82, 1013

Lupton, R., Blanton, M. R., Fekete, G., et al. 2004, PASP, 116, 133

Magrini, L., Vílchez, J. M., Mampaso, A., Corradi, R. L. M., \& Leisy, P. 2007, A\&A, 470, 865

Martin, C. L., \& Kennicutt, Jr., R. C. 2001, ApJ, 555, 301

Mihos, J. C., \& Hernquist, L. 1994a, ApJ, 437, L47

Mihos, J. C., \& Hernquist, L. 1994b, ApJ, 437, 611

Mihos, J. C., \& Hernquist, L. 1996, ApJ, 464, 641

Miller, G. E., \& Scalo, J. M. 1979, ApJS, 41, 513

Miyamoto, M., \& Nagai, R. 1975, PASJ, 27, 533

Naab, T., \& Burkert, A. 2003, ApJ, 597, 893

Naab, T., Jesseit, R., \& Burkert, A. 2006, MNRAS, 372, 839

Ochsenbein, F., Williams, R., Davenhall, C., et al. 2004, in Toward an International Virtual Observatory, ed. P. J. Quinn, \& K. M. Górski, 118

Ocvirk, P., Pichon, C., \& Teyssier, R. 2008, MNRAS, 390, 1326

Ogando, R. L. C., Maia, M. A. G., Chiappini, C., et al. 2005, ApJ, 632, L61

Osuna, P., Barbarisi, I., Salgado, J., \& Laruelo, A. 2008, in Astronomical Spectroscopy and Virtual Observatory, Proceedings of the EURO-VO Workshop, held at the European Space Astronomy Centre of ESA, Villafranca del Castillo, Spain, 21-23 March, 2007, ed. M. Guainazzi, \& P. Osuna, Published by the European Space Agency., 83

Prugniel, P., Soubiran, C., Koleva, M., \& Le Borgne, D. 2007, ArXiv Astrophysics e-prints, (unpublished)

Quinn, P. J., Hernquist, L., \& Fullagar, D. P. 1993, ApJ, 403, 74

Schlegel, D. J., Finkbeiner, D. P., \& Davis, M. 1998, ApJ, 500, 525

Semelin, B., \& Combes, F. 2002, A\&A, 388, 826

Sil'chenko, O. 2006, ApJ, 641, 229

Somerville, R. S., Hopkins, P. F., Cox, T. J., Robertson, B. E., \& Hernquist, L. 2008, MNRAS, 391, 481

Spitzer, L. J., \& Baade, W. 1951, ApJ, 113, 413

Springel, V. 2000, MNRAS, 312, 859

Springel, V., \& Hernquist, L. 2003, MNRAS, 339, 289

Springel, V., Yoshida, N., \& White, S. D. M. 2001, New Astron., 6, 79

Springel, V., Frenk, C. S., \& White, S. D. M. 2006, Nature, 440, 1137

Steinmetz, M., \& Mueller, E. 1994, A\&A, 281, L97

Strateva, I., Ivezić, Ž., Knapp, G. R., et al. 2001, AJ, 122, 1861

Taylor, M., Boch, T., Fitzpartick, M., et al. 2009, IVOA Recommendation, version 1.11, 21 April

Taylor, M. B. 2005, in Astronomical Data Analysis Software and Systems XIV, ed. P. Shopbell, M. Britton, \& R. Ebert, ASP Conf. Ser., 347, 29

Thacker, R. J., Tittley, E. R., Pearce, F. R., Couchman, H. M. P., \& Thomas, P. A. 2000, MNRAS, 319, 619

Toomre, A. 1977, ARA\&A, 15, 437

Toomre, A., \& Toomre, J. 1972, ApJ, 178, 623

Tremonti, C. A., Heckman, T. M., Kauffmann, G., et al. 2004, ApJ, 613, 898

Velazquez, H., \& White, S. D. M. 1999, MNRAS, 304, 254

Walker, I. R., Mihos, J. C., \& Hernquist, L. 1996, ApJ, 460, 121

Wong, T., \& Blitz, L. 2002, ApJ, 569, 157

Zolotukhin, I., \& Chilingarian, I. 2008, in Astronomical Data Analysis Software and Systems XVII, ed. R. W. Argyle, P. S. Bunclark, \& J. R. Lewis, ASP Conf. Ser., 394, 393

Zolotukhin, I., Samokhvalov, N., Bonnarel, F., \& Chilingarian, I. 2007, in Astronomical Data Analysis Software and Systems XVI, ed. R. A. Shaw, F. Hill, \& D. J. Bell, ASP Conf. Ser., 376, 355 